\title{
Executive Dysfunctions and Event-Related Brain Potentials in Patients with Amyotrophic Lateral Sclerosis
}

\author{
Caroline Seer ${ }^{1 * t}$, Stefanie Fürkötter ${ }^{1 \dagger}$, Maj-Britt Vogts ${ }^{1}$, Florian Lange ${ }^{1}$, \\ Susanne Abdulla ${ }^{1,2,3}$, Reinhard Dengler ${ }^{1}$, Susanne Petri ${ }^{1}$ and Bruno Kopp ${ }^{1}$ \\ ${ }^{1}$ Department of Neurology, Hannover Medical School, Hannover, Germany, ${ }^{2}$ Department of Neurology, Otto-von-Guericke \\ University Magdeburg, Magdeburg, Germany, ${ }^{3}$ Department of Neurology, German Center for Neurodegenerative Diseases, \\ Magdeburg, Germany
}

OPEN ACCESS

Edited by:

P. Hemachandra Reddy, Texas Tech University, USA

Reviewed by: Stefano F. Cappa Vita-Salute San Raffaele University,

Italy

Koteswara Rao Valasani, The University of Kansas, USA

*Correspondence: Caroline Seer seer.caroline@mh-hannover.de

${ }^{\dagger}$ These authors have contributed equally to this work and are joint first authors.

Received: 19 August 2015 Accepted: 20 November 2015 Published: 18 December 2015

Citation:

Seer C, Fürkötter S, Vogts M-B, Lange $F$, Abdulla $S$, Dengler $R$, Petri $S$ and Kopp B (2015) Executive Dysfunctions and Event-Related Brain

Potentials in Patients with Amyotrophic Lateral Sclerosis. Front. Aging Neurosci. 7:225 doi: 10.3389/fnagi.2015.00225
A growing body of evidence implies psychological disturbances in amyotrophic lateral sclerosis (ALS). Specifically, executive dysfunctions occur in up to $50 \%$ of ALS patients. The recently shown presence of cytoplasmic aggregates (TDP-43) in ALS patients and in patients with behavioral variants of frontotemporal dementia suggests that these two disease entities form the extremes of a spectrum. The present study aimed at investigating behavioral and electrophysiological indices of conflict processing in patients with ALS. A non-verbal variant of the flanker task demanded two-choice responses to target stimuli that were surrounded by flanker stimuli which either primed the correct response or the alternative response (the latter case representing the conflict situation). Behavioral performance, event-related potentials (ERP), and lateralized readiness potentials (LRP) were analyzed in $21 \mathrm{ALS}$ patients and 20 controls. In addition, relations between these measures and executive dysfunctions were examined. ALS patients performed the flanker task normally, indicating preserved conflict processing. In similar vein, ERP and LRP indices of conflict processing did not differ between groups. However, ALS patients showed enhanced posterior negative ERP waveform deflections, possibly indicating increased modulation of visual processing by frontoparietal networks in ALS. We also found that the presence of executive dysfunctions was associated with more error-prone behavior and enhanced LRP amplitudes in ALS patients, pointing to a prefrontal pathogenesis of executive dysfunctions and to a potential link between prefrontal and motor cortical functional dysregulation in ALS, respectively.

Keywords: amyotrophic lateral sclerosis, ERP, LRP, executive functions, attention

\section{INTRODUCTION}

Amyotrophic lateral sclerosis (ALS) is a devastating neurodegenerative disease characterized by combined degeneration of upper and lower motor neurons. The resulting progressive paralysis involving loss of bulbar and limb muscle function eventually causes death due to respiratory failure within an average of 3 years after symptom onset (Wijesekera and Leigh, 2009). The incidence of ALS in Europe is 2.16 per 100,000 persons (Logroscino et al., 2010), its etiology still remains largely unknown (Strong and Rosenfeld, 2003; Kiernan et al., 2011; Turner et al., 2013). 
While ALS has traditionally been thought to be restricted to the motor nervous system, it is now increasingly acknowledged as a multiple system disease, also affecting non-motor areas of the cortex (Kew et al., 1993a; Abrahams et al., 1996; Geser et al., 2008; Wijesekera and Leigh, 2009; Kiernan et al., 2011; Sarro et al., 2011; Tsermentseli et al., 2012; Agosta et al., 2013; Pettit et al., 2013; Turner et al., 2013; Swinnen and Robberecht, 2014; Turner and Swash, 2015). About 30-50\% of the ALS patients have been reported to develop mild cognitive and behavioral disturbances, with $5-15 \%$ fulfilling the criteria for frontotemporal dementia (FTD; Neary et al., 1998; LomenHoerth et al., 2003; Ringholz et al., 2005; Elamin et al., 2011; Phukan et al., 2012). Behavioral changes accompanying FTD in ALS may involve apathy, personality change, poor insight, and disinhibition, among others (Grossman et al., 2007; Gibbons et al., 2008; Witgert et al., 2010; Abrahams, 2011; Lillo et al., 2011). Cytoplasmic aggregates of the transactive response DNA binding protein (TDP)-43 have been identified in ubiquitinated neuronal inclusion in post mortem brain tissue in ALS patients and in a subgroup of FTD patients, indicating that these disease entities belong to a common disease spectrum (Arai et al., 2006; Neumann et al., 2006; Geser et al., 2008). The overlap of ALS and FTD is further backed up by recent findings in genetics, in particular by the detection of mutations in the TARDP-gene coding for TDP-43 and, more importantly, of hexanucleotide expansions in the C9ORF72 gene as causal for ALS and FTD (DeJesus-Hernandez et al., 2011; Renton et al., 2011; Al-Chalabi et al., 2012; Robberecht and Philips, 2013). Moreover, alterations common to ALS and FTD have been revealed by functional imaging studies. Specifically, neuronal degeneration in prefrontal regions, including dorsolateral prefrontal cortex (dlPFC), and anterior cingulate (ACC), has been linked to some of the cognitive changes of ALS patients (Abrahams et al., 1996, 2005a; Tsermentseli et al., 2012; Pettit et al., 2013). Importantly, not only comorbid FTD (Olney et al., 2005; Elamin et al., 2011) but also executive dysfunction in non-demented ALS patients has been found to be associated with shorter survival times (Elamin et al., 2011).

Non-motor involvement in patients with ALS has been commonly described to manifest in executive dysfunctions (Neary et al., 2000; Phukan et al., 2007; Raaphorst et al., 2010; Goldstein and Abrahams, 2013). Specifically, patients have been found to be impaired in selective attention (Chari et al., 1996; Massman et al., 1996; Abrahams et al., 1997; Pinkhardt et al., 2008; Christidi et al., 2012), verbal fluency (Ludolph et al., 1992; Kew et al., 1993a; Lomen-Hoerth et al., 2003; Massman et al., 1996; Abe et al., 1997; Frank et al., 1997; Rakowicz and Hodges, 1998; Abrahams et al., 2000, 2004, 2005b; Phukan et al., 2012), and cognitive flexibility (Neary et al., 1990; Massman et al., 1996; Abrahams et al., 1997; Moretti et al., 2002; Lange et al., 2015; but see Ludolph et al., 1992; Kew et al., 1993a).

Although non-motor symptoms seem to be common in patients with ALS (Strong et al., 2009), mild cognitive change is difficult to assess in ALS patients due to potential motor and speech deficiencies (Goldstein and Abrahams, 2013). The eventrelated potentials (ERP) technique (Luck, 2005; Raggi et al., 2010) provides an excellent tool to assess cognitive (dys)functions in patients with ALS under minimal motor demands and with high temporal resolution (Neumann and Kotchoubey, 2004; Raggi et al., 2008, 2010; Goldstein and Abrahams, 2013; Lange et al., 2015). ERP waveforms are usually subdivided into early ERP components whose characteristics are associated with physical features of the eliciting stimuli ("exogenous") and late ERP components whose characteristics are associated with cognitive ("endogenous") processing of the stimuli, such as the negativegoing fronto-centrally distributed N2 component (Folstein and van Petten, 2008) and the positive-going parietally distributed P3 component (Polich, 2007). Previous ERP studies revealed that ALS patients show altered endogenous ERP waveforms (Raggi et al., 2010). Specifically, the N2 and P3 latencies were prolonged in ALS patients in perceptual discrimination tasks (Gil et al., 1995; Paulus et al., 2002; Amato et al., 2013). Further, P3 amplitudes were decreased in ALS patients in perceptual discrimination and visual search paradigms (Münte et al., 1999; Hanagasi et al., 2002; Raggi et al., 2008). Contrarily, irrelevant distractor stimuli in an auditory selective attention task were reported to elicit enhanced P3 amplitudes and shortened P3 latencies in ALS patients (Pinkhardt et al., 2008). Taken together, these ERP abnormalities have been interpreted as indices for disturbances in cortical processing for selective attention and executive processing in patients with ALS (Raggi et al., 2010).

Conflict processing represents an important dimension of executive functions (Botvinick et al., 2001). Previous research on conflict processing in ALS mainly focused on the Stroop task, a color-word conflict task, with inconsistent behavioral findings (Ludolph et al., 1992; Kew et al., 1993a; Abrahams et al., 1997; Frank et al., 1997; Thorns et al., 2010; Goldstein et al., 2011; Phukan et al., 2012; Zalonis et al., 2012). The present study aimed to contribute to the literature on executive dysfunctions in ALS by examining electrophysiological correlates of conflict processing. Since language impairments are highly prevalent in ALS patients (Abrahams, 2013; Taylor et al., 2013), we used a non-verbal conflict task for this purpose to avoid potential language confounds (Kopp et al., 1996).

The non-verbal variant of the Eriksen flanker task (Eriksen and Eriksen, 1974) requires the subject to respond to a central target stimulus (e.g., ">”) that is flanked symmetrically by either congruent (">) or incongruent ("<") distractor stimuli (see Rustamov et al., 2013 for discussion). Assessment of response times (RT) and error rates (ER) allows estimating individual information processing abilities, with shorter RT and lower ER indicating more efficient processing. Typically, RT and ER are smaller for congruent situations (e.g., " $>>>$ ") than they are for incongruent situations (e.g., " $><>$ "). This congruency effect (CE) is usually understood as an indicator of conflict processing. In addition, it has been observed that RTs and ERs are not only influenced by the congruency of target and flanker stimuli on any trial, but they are also contextually modulated by the congruency sequence, i.e., by the interaction between the congruency on the current trial and the congruency on the previous trial. Specifically, RT and ER benefits are typically found when a congruent trial was preceded by another congruent trial (this case is labeled $c C$ trial throughout this paper) rather than by an incongruent trial $(i C)$, and when an incongruent 
trial was preceded by another incongruent trial (iI) rather than by a congruent trial $(c I)$. In the flanker task, this congruency sequence effect (CSE; Gratton et al., 1992; Egner, 2007) has been found to occur specifically when the response can be repeated across two successive trials, but to be completely absent when responses alternate across two successive trials (Rustamov et al., 2013). Figure 1 depicts the trial sequences of interest (cC, iC, iI, cI) separately for response repetition and response alternation trials. Inspection of Figure 1 reveals that $c C$ and $i I$ trials enable repetition priming (via exact stimulus repetitions) in response repetition, but not in response alternation trials. The specificity of the CSE for response repetition trials suggests that priming plays an important role in contextual modulation of conflict processing as assessed by the flanker task (Mayr et al., 2003). Until now, no study investigated conflict processing and its contextual modulation in ALS by means of the flanker task. However, Luks et al. (2010) found the CE in error rates (i.e., more errors in incongruent trials than in congruent trials) on the flanker task to be related to atrophy of the dlPFC and ACCi.e., two prefrontal regions that have been related to the presence of cognitive impairments in patients with ALS (Abrahams et al., 1996; Goldstein et al., 2011; Tsermentseli et al., 2012; Pettit et al., 2013).

The ERP technique offers a fine-grained analysis of the cognitive processes as they occur in rapid succession during information processing. To begin with, posterior negative ERP waveform deflections indicate modulation of visual processing by frontoparietal attention networks (Ptak, 2012; Rustamov et al., 2014; Vossel et al., 2014). For example, selective attention to relevant stimulus features is typically associated with the appearance of a selection negativity $(\mathrm{SN})$ at posterior electrode sites. The $\mathrm{SN}$ is a negative ERP waveform deflection that has its onset at around $160 \mathrm{~ms}$ post-stimulus and persists for approximately $200 \mathrm{~ms}$ and is thought to indicate prioritized processing of attended stimulus features (Hillyard and AnlloVento, 1998; Hillyard et al., 1998; Kopp et al., 2007; Kopp and Wessel, 2010). Compelling evidence that posterior negativities are subject to prefrontal modulation comes from a study of patients with unilateral focal prefrontal lesions. This study revealed that prefrontal damage reduced posterior negativities, putatively originating from neuronal activity in the extrastriate cortex of the lesioned hemisphere, during visual discrimination (Barceló et al., 2000).

The fronto-centrally distributed N2 component of the ERP is a negative-going potential that is typically observed around 250-300 ms post-stimulus. The N2 has been proposed to reflect conflict processing (Folstein and van Petten, 2008). Correspondingly, N2 amplitudes are typically enhanced on incongruent trials (i.e., stimuli are associated with a higher degree of conflict) of the Eriksen flanker task (Kopp et al., 1996; Yeung et al., 2004; Danielmeier et al., 2009). Likewise, the CSE has been associated with N2 modulations such that enhanced $\mathrm{N} 2$ amplitudes are observed on incongruent trials preceded by congruent trials $(c I)$ than on incongruent trials preceded by incongruent trials (iI; Clayson and Larson, 2011a,b, 2012; Freitas et al., 2009; Forster et al., 2011; Larson et al., 2012; Rustamov et al., 2013). Interestingly, the individual differences with regard to the degree of these CSE-related N2 modulations correlate with cognitive performance in tests of attention, executive functions and verbal fluency (Clayson and Larson, 2012). In a recent study, Rustamov et al. (2013) found that N2 amplitudes were modulated by the congruency sequence in control participants, but the CSE-related N2 modulation was absent in patients with Parkinson's disease (PD).

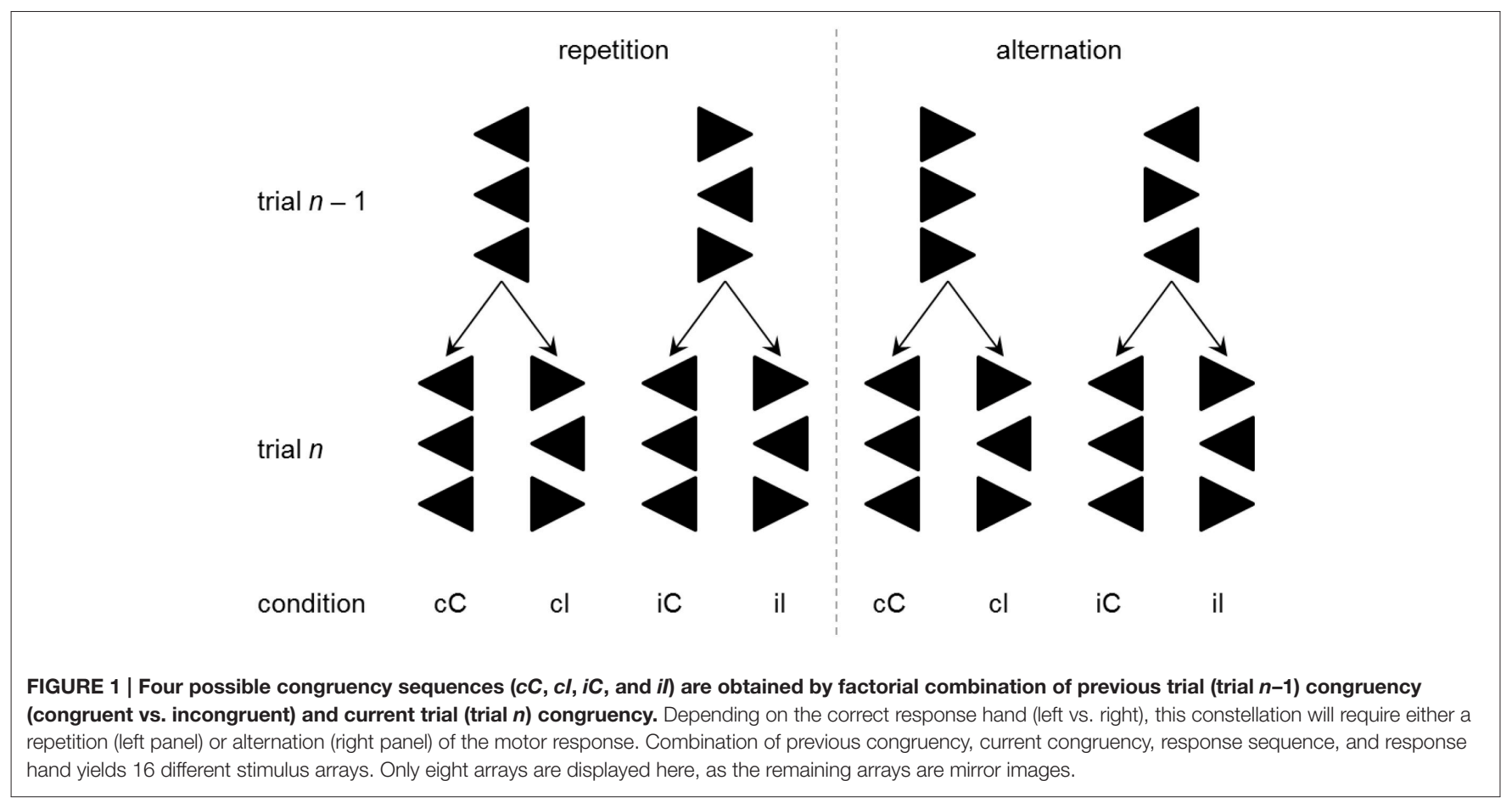


The lateralized readiness potential (LRP; Coles, 1989; Eimer, 1998) has its neural generators in primary motor areas (Leuthold and Jentzsch, 2002) and it indexes the duration of the selection and of the preparation of appropriate motor responses. In the context of tasks requiring uni-manual responses, recordings from two electrodes over the primary motor areas can be used to calculate the LRP such that negative deflections indicate the activation and preparation of a uni-manual correct response. The stimulus-locked LRP (s-LRP) onset latency indicates the time interval between stimulus onset and the initiation of response preparation. Thus, s-LRP onset latencies provide a measure of the duration of pre-motor (perceptual and cognitive) processes (i.e., stimulus encoding plus response selection). In the context of the Eriksen flanker task, s-LRP onset latencies revealed that the preparation of the correct response started earlier on congruent trials than it did on incongruent trials (Gratton et al., 1988; Kopp et al., 1996).

Finally, LRP amplitudes provide measures of the functional dysregulation of cortical motor-generation processes. For example, normal aging is associated with an enhancement of LRP amplitudes (Yordanova et al., 2004; Roggeveen et al., 2007; Wild-Wall et al., 2008; Vallesi and Stuss, 2010; Cespón et al., 2013; Cid-Fernández et al., 2014). There is one published study which examined LRP amplitudes in ALS patients, and it reported diminished LRP amplitudes in a response inhibition paradigm (Thorns et al., 2010).

Here, we used a combination of behavioral and electrophysiological measures (ERP, LRP) obtained on the flanker task to examine conflict processing (CE) and its contextual modulation (CSE) in ALS patients. Several hypotheses about potentially distinct cognitive impairments are conceivable. Some of them are not mutually exclusive; nevertheless, they allow to study the dynamic interplay between selective attention, conflict processing and motor preparation. First, ALS patients may suffer from disturbed attentional modulation of visual processing (Chari et al., 1996; Massman et al., 1996; Abrahams et al., 1997; Pinkhardt et al., 2008; Christidi et al., 2012) that should express itself in altered posterior negativities. A corollary of such an attentional disturbance would be that flanker stimuli impose increased levels of conflict in ALS patients compared to controls, leading to enhanced behavioral (i.e., larger RT and ER differences between congruent and incongruent trials) and neural (i.e., disproportionally increased N2 amplitudes and prolonged s-LRP onset latencies on incongruent trials) indicators of conflict processing. Another possibility is that the cognitive disturbances in ALS are confined to impaired contextual modulation of conflict processing. This hypothesis leads to the prediction of altered behavioral and neural indicators of the CSE, as discussed above and as demonstrated in an earlier study from our group in patients with PD (Rustamov et al., 2013). Finally, ALS patients may show dysregulated cortical motor preparation processes, and this dysregulation should manifest itself in altered LRP amplitudes. We also assessed participants' performance on a number of neuropsychological tests in order to evaluate potential relationships between clinically manifest indicators of executive dysfunctions in ALS patients and behavioral and neural indices of conflict processing and its contextual modulation.

\section{METHODS}

\section{Participants}

A cohort of 21 patients with ALS [15 males (71.4\%); age: $M=$ 58.90 years, $S D=9.62$, range: $34-76$; education years: $M=$ 13.86 years, $S D=2.29$, range: $10-19$ ] was recruited between July 2013 and January 2014 from Hannover Medical School. Patients were included if they fulfilled the revised El Escorial criteria for clinically probable or definite ALS (Brooks et al., 2000). Eighteen patients had limb-onset disease, whereas three patients had bulbar-onset disease, and one patient fulfilled the criteria for FTD (Neary et al., 1998). Four patients were treated with nocturnal non-invasive ventilation (NIV) and none of the patients had a percutaneous endoscopic gastrostomy (PEG). A third-party rating (Abrahams et al., 2014) was administered to the patients' relatives or caregivers to assess potential behavioral alterations and psychotic symptoms. This information was available for 12 patients, and indicated that none of the patients showed behavioral disinhibition, whereas apathy and hyperorality/altered eating behavior were reported in two cases, and loss of sympathy/empathy and perseverative/stereotyped behavior were reported in three cases. One patient was reported to show psychotic tendencies. Criteria for exclusion involved other neurological diseases, any psychiatric disorder, and highly restricted pulmonary function. Furthermore, patients were excluded when they were too impaired to press a button of a keyboard due to their disease severity. Clinical status of ALS patients was investigated using the ALSFRS-EX (Abdulla et al., 2013; see Table 1), a 15-item self-report measure assessing functional impairments, which is an adapted and validated version of the well-established revised Amyotrophic Lateral Sclerosis Functional Rating Scale (ALSFRS-R; Cedarbaum et al., 1999). Respiratory function was quantified by the forced vital capacity (FVC), revealing an average FVC of 85.47 ( $S D=14.62$, range: 60-109). Additionally, daytime sleepiness was assessed using the Epworth Sleepiness Scale (ESS; Johns, 1991), revealing an average of $5.55(S D=3.17$, range: $0-11)$. Twenty-one age-, gender-, and education-matched healthy (i.e., these participants were not diagnosed with ALS nor with any other neurological disease) controls (HC) were examined [age: $M=57.67$ years, $S D=9.16$, range 44-74 years; 15 males (71.4\%); education years: $M=14.29$ years, $S D=2.93$, range $=10.5-20.5]$. The HC group did not differ from ALS patients with regard to age, $t_{(40)}=-0.43, p=0.672$, or education years, $t_{(40)}=0.53$, $p=0.600$. Control participants were either spouses or friends of the participants, or volunteers who were compensated for their participation with payment $(30 €)$. One control participant had to be excluded from the analyses regarding the flanker task due to poor task comprehension. All participants were righthanded and had normal or corrected-to-normal vision and intact hearing. The study was reviewed and approved by the local ethics committee (Ethics Committee of Hannover Medical School: vote number 6269). All participants gave written informed consent in accordance with the Declaration of Helsinki.

A battery of neuropsychological tests was administered to the participants. The Edinburgh Cognitive and Behavioral ALS Screen (ECAS; Abrahams et al., 2014) was used to examine 
TABLE 1 | Summary statistics on clinical characteristics and neuropsychological measures for ALS patients (ALS) and controls (HC) and ALS patients with (ALSef-) and without (ALSef+) executive dysfunctions according to the M-WCST EFC.

\begin{tabular}{|c|c|c|c|c|c|c|c|}
\hline & $\max$. & \multicolumn{2}{|c|}{ Mean $(S D)$} & $p^{\mathrm{a}}$ & \multicolumn{2}{|c|}{ Mean $(S D)$} & $p^{\mathbf{b}}$ \\
\hline ALSFRS-EX total & 60 & & $46.85(7.18)$ & & $47.20(5.61)$ & $46.50(8.77)$ & 0.834 \\
\hline Bulbar subscore & 16 & & $13.65(1.98)$ & & $13.50(2.12)$ & $13.80(1.93)$ & 0.745 \\
\hline Fine motor subscore & 16 & & $11.45(2.91)$ & & $12.30(3.02)$ & $10.60(2.67)$ & 0.199 \\
\hline Respiratory subscore & 12 & & $10.90(1.74)$ & & $11.40(1.26)$ & $10.40(2.07)$ & 0.208 \\
\hline Progression rate & & & $0.75(0.54)$ & & $0.80(0.55)$ & $0.69(0.55)$ & 0.667 \\
\hline ECAS total & 136 & $104.38(11.58)$ & $102.05(13.12)$ & 0.545 & $97.50(15.02)$ & $104.60(9.08)$ & 0.217 \\
\hline ECAS ALS-specific & 100 & $75.48(10.63)$ & $74.10(11.22)$ & 0.684 & $68.70(12.36)$ & $77.90(7.09)$ & 0.056 \\
\hline Language & 28 & $26.62(1.94)$ & $26.14(2.06)$ & 0.444 & $26.20(2.15)$ & $26.00(2.16)$ & 0.838 \\
\hline Visuospatial & 12 & $11.62(0.74)$ & $11.48(0.68)$ & 0.518 & $11.40(0.70)$ & $11.60(0.70)$ & 0.530 \\
\hline FAB & 18 & $17.19(1.25)$ & $16.37(2.19)$ & 0.162 & $16.10(2.33)$ & $16.5(2.20)$ & 0.716 \\
\hline M-WCST EFC & & $106.81(15.66)$ & $96.15(21.50)$ & 0.079 & $77.70(13.03)$ & $114.60(7.07)$ & $<0.001$ \\
\hline Categories & 7 & $5.76(1.55)$ & $4.65(2.16)$ & 0.068 & $3.00(1.89)$ & $6.30(0.48)$ & $<0.001$ \\
\hline Perseveration errors & 47 & $1.33(2.74)$ & $3.40(5.02)$ & 0.115 & $6.60(5.50)$ & $0.20(0.42)$ & 0.005 \\
\hline MoCA & 30 & $27.24(2.68)$ & $26.62(3.06)$ & 0.488 & $25.60(3.13)$ & $27.50(2.95)$ & 0.181 \\
\hline
\end{tabular}

ECAS, Edinburgh Cognitive and Behavioral ALS Screen; FAB, Frontal Assessment Battery; M-WCST, Modified Wisconsin Card Sorting Test; EFC, Executive Function Composite; MoCA, Montreal Cognitive Assessment.

${ }^{a} H C$ vS. ALS.

${ }^{b} A L S e f-v s . A L S e f+$.

ALS-specific (executive functions, verbal fluency, language) as well as non-ALS-specific (memory, visuospatial functions) cognitive abilities. Frontal lobe functions were examined using the Frontal Assessment Battery (FAB; Dubois et al., 2000; Kopp et al., 2013). Participants were screened for mild cognitive impairment and dementia using the Montreal Cognitive Assessment (MoCA; Nasreddine et al., 2005).

To further assess potential effects of clinically manifest executive dysfunctions, we compared patients with relatively high executive performance to those with a relatively poor performance. To this end, we used individual Executive Function Composite (EFC) scores that can be obtained from the Modified Wisconsin Card Sorting Test (M-WCST; Schretlen, 2010). The M-WCST-based EFC represents a global measure of executive functions which is derived from the number of categories and from the number of perseverative errors on a newly standardized analog of the well-known WCST (Heaton et al., 1993). As this score could be obtained from 20 ALS patients, we established two equally-sized subgroups of ALS patients on the basis of this EFC with a population mean of 100 and a population standard deviation of 15 via a median split. The subgroup that showed below-median performance (ALSef-) showed "borderline" executive dysfunctions on average $(M=$ 77.07; $S D=13.03$; i.e., around $1.53 \mathrm{SDs}$ below the population mean), while the other subgroup (ALSef + ) performed at "high average" on the M-WCST ( $M=114.60 ; S D=7.07$; i.e., around
0.97 SDs above the population mean). Thus, the ALS subgroups differed from each other on the EFC of the M-WCST as expected, but at unforeseen sharpness (by around $2.5 \mathrm{SDs}$ ), illustrating the heterogeneity of executive functions in ALS patients. The ALSefand ALSef + subgroups did not differ with regard to their mean age (ALSef-: $M=63.30, S D=8.26$; ALSef+: $M=55.20, S D=$ 9.76; $p=0.061$ ) or education years (ALSef-: $M=13.55, S D=$ 1.67; ALSef+: $M=13.65, S D=2.39 ; p=0.915$ ). The ALSef + and ALSef- group did not differ with regard to other clinical characteristics, except for the ECAS ALS-specific executive score (ECAS EFS; ALSef-: $M=34.10, S D=8.66$; ALSef $+: M=40.90$, $S D=3.25 ; p=0.039$ ), confirming the differences in executive functions revealed by the M-WCST (Table 1). Figure 2 displays histograms of M-WCST EFC and ECAS EFS scores, separately for ALS patients and HC. Inspection reveals that the M-WCST EFC scores sub-divided ALS patients into two, roughly equally sized subgroups (ALSef- and ALSef+, respectively).

\section{Materials and Procedure}

Stimulus material was run by Presentation ${ }^{\circledR}$ (Neurobehavioral Systems, Albany, CA). Stimuli were presented for $300 \mathrm{~ms}$ against a black background on a computer screen (Eizo EV2416W, Eizo, Hakusan, Japan), subtending a visual angle of $4^{\circ} \times$ $1^{\circ}$ at a viewing distance of $1.45 \mathrm{~m}$. Each stimulus consisted of three white arrows pointing to the left or to the right (Figure 1). Arrows were arranged vertically such that the two 

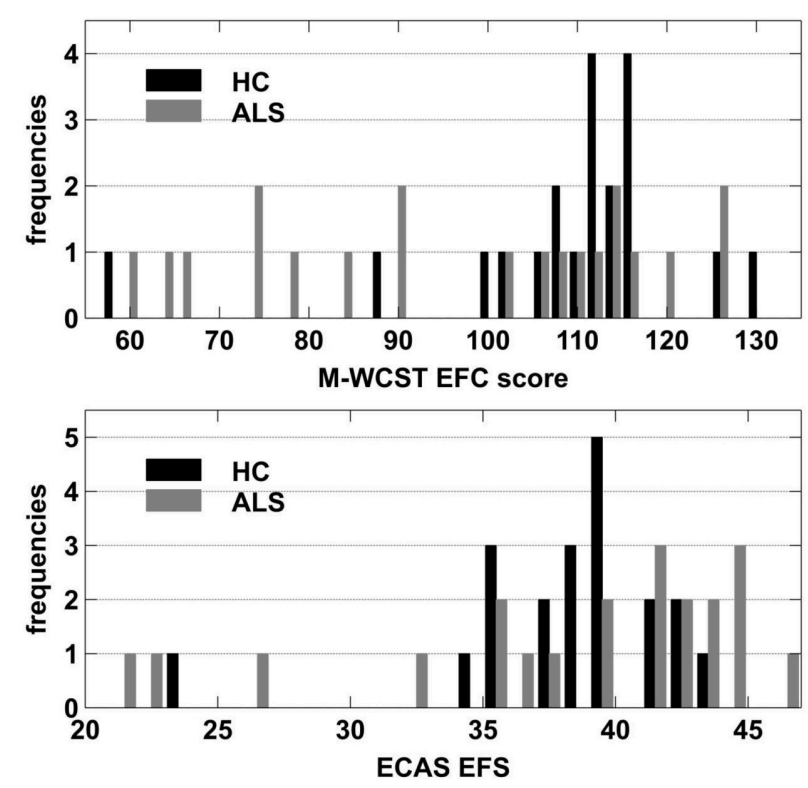

FIGURE 2 | Frequency histograms of M-WCST EFC scores (upper panel) and ECAS EFS (lower panel) separately for ALS patients and HC.

outer arrows ("flanker arrows") either pointed to the same (congruent) or to the opposite (incongruent) direction as the central arrow ("target arrow"). Participants were informed that on each trial, three arrows would appear on the computer screen, and that their task was to focus on the central arrow which would either point to the left or to the right, while ignoring the flanking arrows. They were asked to press the left or right Ctrl key on a standard computer keyboard when the central target arrow pointed to the left or right, respectively. Varying the direction of the target arrow (left vs. right) and the congruency of the stimulus array (congruent vs. incongruent) generated four different flanker-target combinations (congruentleft, congruent-right, incongruent-left, incongruent-right). As the direction factor was not of interest for the analyses in this study, congruent-left and congruent-right as well as incongruentleft and incongruent-right stimuli were grouped, and this factor is ignored in the following. Hence, two types of trials are distinguishable: on congruent trials, flanker and target arrows are associated with the same motor response whereas on incongruent trials, flanker and target arrows are associated with contradicting motor responses. Comparing the trial types of the current trial (congruent vs. incongruent) and the type of the respective preceding trial (congruent vs. incongruent) yields four different congruency sequences: preceding trial congruentcurrent trial congruent $(c C)$, preceding trial congruent-current trial incongruent $(c I)$, preceding trial incongruent-current trial congruent $(i C)$, and preceding trial incongruent-current trial incongruent $(i I)$. The response sequence indicates whether the motor response on the current trial had to be repeated (e.g., the target arrow points to the left both on the current trial and the previous trial) or altered (e.g., the target arrow points to the left on the current trial, but had pointed to the right on the previous trial) with respect to the previous trial. Factorial combination of the response sequence with the congruency sequence yields eight experimental conditions ( $c C$-repetition, $c C$-alternation, $c I$-repetition, $c I$-alternation, $i C$-repetition, $i C$-alternation, $i I-$ repetition, and $i I$-alternation). The distinction between response sequences allows for determining whether the CSE is specific to response repetition trials, as described in the Introduction.

Four hundred and thirty-two (50\% congruent, 50\% incongruent) stimuli were presented in four blocks in randomized order with a response-stimulus interval of $2000 \mathrm{~ms}$. Response repetition and response alternation trials were equally probable in our task. To acquaint participants to the task, 12 practice trials were presented prior to the experimental phase.

\section{Electrophysiological Recording}

Continuous electroencephalogram was recorded with a 32channel BrainAmp amplifier (Brain Products, Gilching, Germany) and active Ag-AgCl electrodes (Brain Products, Gilching, Germany) mounted on an actiCap (EASYCAP, Herrsching, Germany) according to the international 10-20 system montage. BrainVision Recorder software (Brain Products, Gilching, Germany) was used. Electrode impedance was kept below $10 \mathrm{k} \Omega$. Electrodes were referenced to $\mathrm{FCz}$ electrode. To monitor ocular artifacts, vertical (vEOG), and horizontal (hEOG) electrooculogram were recorded with two electrodes positioned at the suborbital ridge and the external ocular canthus of the right eye, respectively.

\section{Data Analysis \\ Behavioral Data}

RTs and ERs were calculated with respect to the congruency on the current trial and the congruency sequence separately for response repetition and response alternation trials. RTs were obtained by computing the median response latency on correctly completed trials that occurred between 100 and $2000 \mathrm{~ms}$ after stimulus onset. ERs were calculated as the proportion of erroneous responses.

\section{Electrophysiological Data}

EEG data were evaluated using BrainVision Analyzer 2.0 (Brain Products, Gilching, Germany). After bandpass filtering (highpass: $0.5 \mathrm{~Hz}, 24 \mathrm{~dB} /$ oct; low-pass: $70 \mathrm{~Hz}, 24 \mathrm{~dB} /$ oct; notch: $50 \mathrm{~Hz}$ ), data were screened for artifacts (max. allowed voltage step: $75 \mu \mathrm{V} / \mathrm{ms}$; lowest allowed activity: $0.5 \mu \mathrm{V} / 100 \mathrm{~ms}$ ), and subjected to an ocular-correction independent component analysis (ICA; Groppe et al., 2008) for further removal of ocular, muscular, and cardiac artifacts. For every possible combination of congruency and response sequence and separately for left and right hand responses, data of correctly completed trials were then segmented into epochs of $1200 \mathrm{~ms}$ relative to stimulus onset, baseline corrected (baseline: $-200-0 \mathrm{~ms}$ ), and underwent an artifact rejection procedure [max. allowed voltage difference: $150 \mu \mathrm{V} / 200 \mathrm{~ms}$; max. allowed amplitude: $-100 \mu \mathrm{V}(\min ), 100 \mu \mathrm{V}$ $(\max )]$ approved by careful visual inspection in order to exclude remaining artifacts. Data were averaged and re-referenced to a common average reference. 
ERP and LRP waves were analyzed for trials that were correctly completed between 100 and $2000 \mathrm{~ms}$ after stimulus onset. For the examination of attention-related ERP, we compared posterior negativity amplitudes between ALS patients and HC. As we were primarily interested in differences between ALS patients and HC, we compared ERP waves occurring in the latency area of attention-related posterior negativities between these groups in order to determine potential alterations in attentional processing in ALS patients. Specifically, posterior negativities were calculated as mean amplitudes at $\mathrm{O} 1$ and $\mathrm{O} 2$ electrodes in the time window from -60 to $+60 \mathrm{~ms}$ around $196 \mathrm{~ms}$ after stimulus onset, where the difference wave of HC-ALS patients reached its maximum at occipital sites. For the analysis of frontocentral N2 waves, peaks were identified in single-subject averages on $\mathrm{Cz}$ electrode within a latency area of 200 and $320 \mathrm{~ms}$ after stimulus onset (Kopp et al., 1996). The N2 was then calculated as the mean amplitude in the time window from -60 to $+60 \mathrm{~ms}$ of the individual N2 peak latency. The stimulus-locked LRP (sLRP) was obtained by subtracting signals recorded at ipsilateral sites (e.g., C3 or C4) from the signals at contralateral sites (e.g., C4 or C3, respectively) separately for the hands, and averaging these difference waves for every subject and condition. s-LRP epochs were created relative to stimulus onset (from $-200 \mathrm{~ms}$ preceding the stimulus to $1000 \mathrm{~ms}$ post-stimulus) separately for all possible combinations of congruency sequence and response sequence for correctly completed trials. Furthermore, the response-locked LRP (LRP-r) was calculated in order to examine the processes before response execution. LRP-r epochs comprised the $1000 \mathrm{~ms}$ preceding the motor response as well as the following $350 \mathrm{~ms}$ (Eder et al., 2012). LRP onset differences were evaluated in low-pass filtered data $(5 \mathrm{~Hz}, 12 \mathrm{~dB} / \mathrm{oct})$ applying the jackknifing method as described in Miller et al. (1998) and Ulrich and Miller (2001; see also Eder et al., 2012) that is a suitable technique for the determination of latency differences (Kiesel et al., 2008). Briefly, grand averages are repeatedly calculated for every condition, every time omitting one participant. By this means, changes in LRP onsets can be determined in each of the resulting grand averages with a higher signal-to-noise ratio than achieved in conventional analyses. We defined the LRP onset as the point in time where the LRP amplitude first reached $50 \%$ of its maximum amplitude (i.e., the maximum amplitude in that condition including all participants), as proposed by Miller et al. (1998; see also Eder et al., 2012). The resulting values are then subjected to an ANOVA. As this method further underestimates the actual between-subjects variance, $F$-values need to be corrected so that $F_{c}=F /(n-1)^{2}$ (Miller et al., 1998; Ulrich and Miller, 2001). $F_{c}$ is then compared to the critical $F$-values. As EEG data obtained from $21 \mathrm{ALS}$ patients and 20 $\mathrm{HC}$ were analyzed, but equal sample sizes are required for the comparison of jackknifed data described here, we excluded the patient with the noisiest EEG from the LRP onset analyses (Ulrich and Miller, 2001). LRP amplitudes were measured as individual peak minima at the $\mathrm{C} 3-\mathrm{C} 4$ electrode pair after $5-\mathrm{Hz}(12 \mathrm{~dB} / \mathrm{oct})$ low-pass filtering.

For statistical analyses, repeated measurement ANOVA was used with group (ALS vs. HC) as between-subject factor and conditions (congruency on current trial: congruent vs. incongruent; congruency on previous trial: congruent vs. incongruent; response sequence: repetition vs. alternation) as within-subject factors. For the analyses of posterior negativity amplitudes, the factor electrode site (O1 vs. O2) was added and the factors congruency on previous trial and response sequence were not included as these effects were not of interest for the present study. Effect sizes for ANOVA results were calculated as $\eta_{p}^{2}$. Greenhouse-Geisser corrections were used where appropriate. For post-hoc tests, $p$-values are reported after Bonferroni correction for multiple comparisons. For correlation analyses, Spearman-Brown correlation coefficients were calculated. To determine potential associations between executive performance, task performance and electrophysiological measures, we correlated the EFC of the M-WCST and the ECAS EFS with RT, ER and the amplitudes of the posterior negativity, N2, s-LRP and LRP-r, each averaged across all conditions of current congruency, previous congruency and response sequence. The posterior negativity amplitudes were averaged over $\mathrm{O} 1$ and $\mathrm{O} 2$ electrodes for the correlation analyses.

\section{RESULTS}

\section{Neuropsychological Tests}

The results obtained from neuropsychological testing are displayed in Table 1. ALS patients did not show statistically significant impairments in performance in comparison to $\mathrm{HC}$ in any of the tests that were applied.

This study brought into focus whether flanker congruency (i.e., CE) and/or congruency sequence (i.e., CSE) exerted differential effects on behavioral, ERP and LRP responses in ALS patients and HC. The results thus primarily report the effects of group, of flanker congruency (i.e., CE), of the congruency sequence by response sequence interaction (i.e., CSE by Response Sequence), as well as the interactions between group and these two repeated-measures manipulations.

\section{Behavioral Data \\ Response Times}

As shown in Figure 3, ALS patients (mean RT $=593 \mathrm{~ms}$ ) responded slower than $\mathrm{HC}$ (mean $\mathrm{RT}=546 \mathrm{~ms}$ ); however, this apparent response slowing did not reach statistical significance, $F_{(1,39)}=2.60, p=0.115, \eta_{p}^{2}=0.063$. There was a significant CE, $F_{(1,39)}=192.19, p<0.001, \eta_{p}^{2}=0.831$, and a significant CSE by Response Sequence interaction, $F_{(1,39)}=56.27, p<0.001$, $\eta_{p}^{2}=0.591$, indicating that these independent manipulations exerted massive effects on RT variation. As expected, congruent trials showed faster mean RT $(536 \mathrm{~ms})$ than incongruent trials (604 ms). On response repetition trials (cf. Figure 1), mean RT on $c C$ and $i I$ trials amounted to $528 \mathrm{~ms}$ and $599 \mathrm{~ms}$, respectively, which is shorter than those on $i C$ and $c I$ trials $(541 \mathrm{~ms}$ and $639 \mathrm{~ms}$ ), compatible with the expected CSE on these trials. On response alternation trials (cf. Figure 1), mean RT on $c C$ and $i I$ trials amounted to 536 and $592 \mathrm{~ms}$, respectively, which is roughly identical to those on $i C$ and $c I$ trials (539 and $586 \mathrm{~ms}$ ), compatible with the expected absence of the CSE on these trials. Group did 


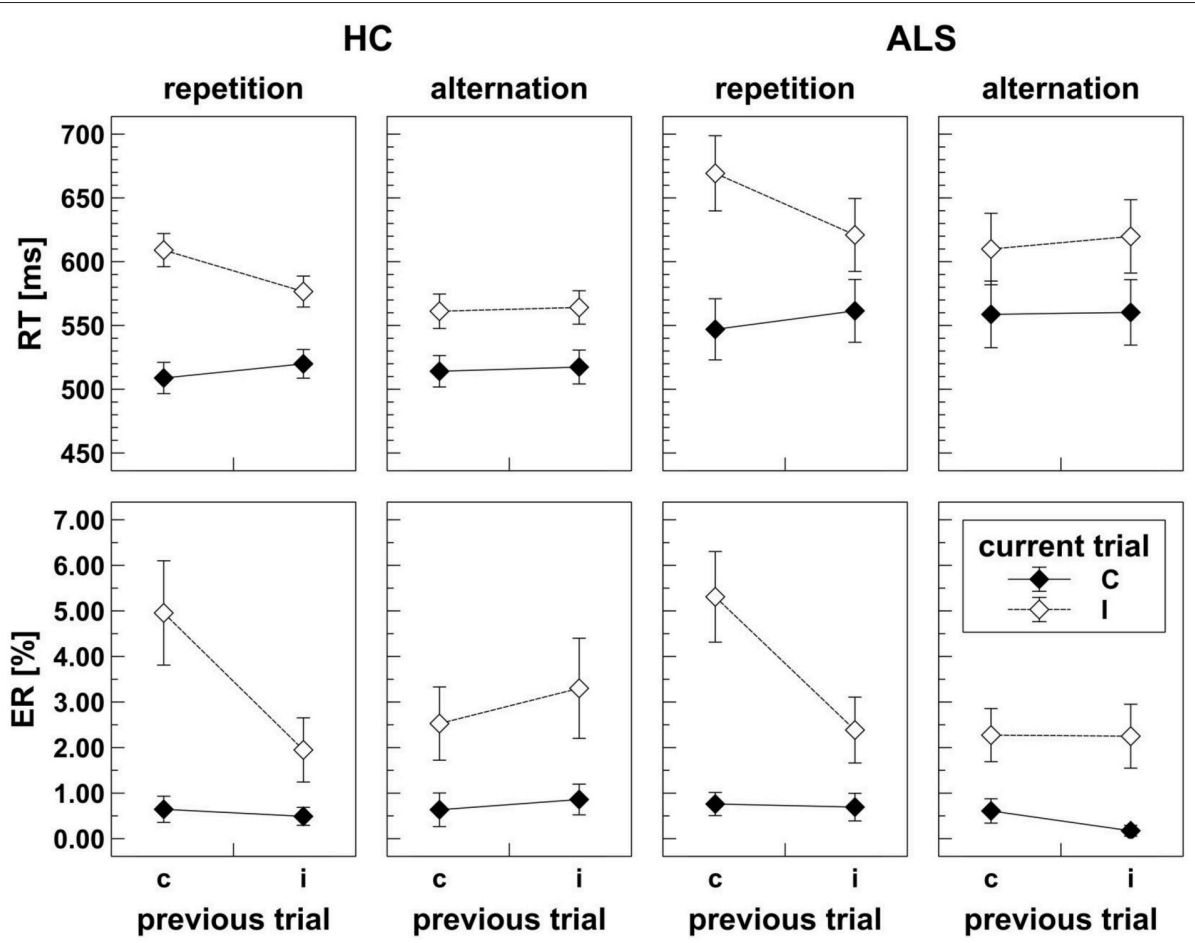

FIGURE 3 | Behavioral data obtained in the flanker task for HC (left panels) and ALS patients (right panels). Upper panels display response times (RT) separately for response repetition and response alternation trials. Lower panels display error rates (ER) separately for response repetition and response alternation trials. Lower case $c$ (congruent) and $i$ (incongruent) on the abscissa indicate previous trial congruency level; upper case $C$ (congruent) and I (incongruent) on separate lines indicate current trial congruency level. For HC as well as for ALS patients, response times and error rates are modulated by the typical effects of congruency and a congruency sequence effect that is present in response repetition, but absent in response alternation trials.

not significantly modulate the CE, $F_{(1,39)}=1.14, p=0.292$, $\eta_{p}^{2}=0.028$, nor the CSE by Response Sequence interaction, $F_{(1,39)}=3.34, p=0.075, \eta_{p}^{2}=0.079$.

\section{Error Rates}

Visual inspection revealed that error data were not normally distributed. ANOVA results did not change when we repeated the analyses with arcsine-transformed or log-transformed $(y=$ $\log (1.1-x))$ data. For simplicity, we report the results of untransformed ER data here. ER were similar for ALS patients (mean ER $=1.8 \%$ ) and $\mathrm{HC}$ (mean ER $=1.9 \%$ ), such that the main effect of Group did not reach statistical significance, $F_{(1,39)}=$ 0.04, $p=0.846, \eta_{p}^{2}=0.001$. There was a significant $\mathrm{CE}$, $F_{(1,39)}=40.98, p<0.001, \eta_{p}^{2}=0.512$, and a significant CSE by Response Sequence interaction, $F_{(1,39)}=17.75, p<0.001$, $\eta_{p}^{2}=0.313$, indicating that these manipulations exerted strong effects on ER variation. As expected, fewer errors were committed on congruent trials $(0.6 \%)$ than on incongruent trials $(3.1 \%)$. On response repetition trials (cf. Figure 1), ER on $c C$ and $i I$ trials amounted to 0.7 and $2.2 \%$, respectively, whereas ER on $i C$ and $c I$ trials were 0.6 and $5.1 \%$, compatible with the expected CSE on these trials. On response alternation trials (cf. Figure 1), ER on $c C$ and iI trials amounted to 0.6 and $2.8 \%$, respectively, which is roughly identical to those on $i C$ and $c I$ trials ( 0.5 and $2.4 \%)$, compatible with the expected absence of the CSE on these trials. Group did not significantly modulate CE nor the CSE by Response Sequence interaction (all $F<0.01$, all $p>0.05$ ).

\section{Electrophysiological Data Posterior Negativity}

Figure 4 displays posterior negativities that were recorded from ALS patients and HC. ALS patients $(-3.69 \mu \mathrm{V})$ showed enhanced amplitudes compared to $\mathrm{HC}(-1.43 \mu \mathrm{V})$, as reflected in a statistically significant main effect of Group, $F_{(1,39)}=6.98, p=$ $0.012, \eta_{p}^{2}=0.152$. There was no main effect of CE, $F_{(1,39)}=0.08$, $p=0.782, \eta_{p}^{2}=0.002$, and the Group by CE interaction failed to reach statistical significance, $F_{(1,39)}=3.25, p=0.079, \eta_{p}^{2}=$ 0.077 .

\section{N2}

As shown in Figure 5, N2 amplitudes in ALS patients $(-0.53 \mu \mathrm{V})$ and in $\mathrm{HC}(-0.92 \mu \mathrm{V})$ achieved similar levels. Group, CE, and the CSE by Response Sequence interaction as well as interactions between Group and CE or CSE by Response Sequence were not statistically significant (all $F<1.84$ all $p>0.05$ ). However, N2 amplitude was modulated by the congruency of the trial preceding the current trial, $F_{(1,39)}=5.19, p=0.028, \eta_{p}^{2}=0.117$, such that $\mathrm{N} 2$ amplitudes were more negative when the preceding trial was incongruent $(-0.79 \mu \mathrm{V})$ than when the preceding trial was congruent $(-0.67 \mu \mathrm{V})$. 


\section{s-LRP Onset Latencies}

Inspection of Figure 6 reveals that s-LRP onset latencies were strongly modulated by CE, $F_{c}(1,38)=53.51, p<0.001, \eta_{p}^{2}=$ 0.585 , with shorter latencies on congruent $(287 \mathrm{~ms})$ compared to incongruent trials $(370 \mathrm{~ms})$. The overall effect of $\mathrm{CE}$ was further modulated by Response Sequence, $F_{c(1,38)}=5.99$, $p=0.019, \eta_{p}^{2}=0.136$, indicating that onset latencies on congruent trials were shorter on response repetition $(282 \mathrm{~ms})$ than on response alternation trials $(291 \mathrm{~ms})$, whereas onset latencies on incongruent trials were shorter on response alternation $(351 \mathrm{~ms})$ than on response repetition trials $(388 \mathrm{~ms})$. Group, alone or in interaction with CE or CSE by Response Sequence, did not reach statistical significance (all $F<0.23$, all $p>0.05$ ).
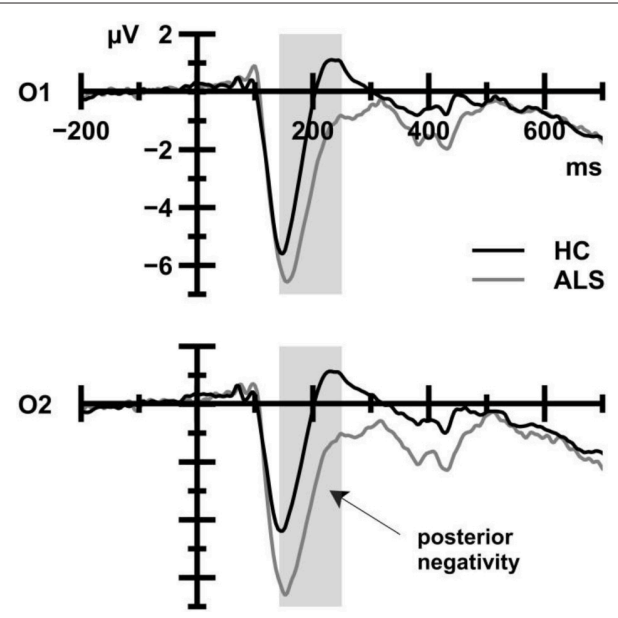

FIGURE 4 | ERP waveforms that were obtained from HC (black) and ALS patients (gray) averaged over all trial types. The shaded area indicates the time window for analyses. ALS patients show more pronounced posterior negativities at occipital sites compared to $\mathrm{HC}$.

\section{LRP Amplitudes}

$s-L R P$

Figure 7 (left panel) displays s-LRP amplitudes that were recorded from ALS patients and HC. In short, s-LRP amplitudes were unaffected by Group, either alone or in interaction with $\mathrm{CE}$ and the CSE by Response Sequence interaction (all $F<1.80$, all $p>0.05)$. However, s-LRP amplitudes were enhanced on response alternation $(-3.43 \mu \mathrm{V})$ compared to response repetition trials $(-2.86 \mu \mathrm{V})$, giving rise to a statistically significant Response Sequence effect, $F_{(1,39)}=32.07, p<0.001$, $\eta_{p}^{2}=0.451$.

\section{LRP-r}

Figure 7 (right panel) displays LRP-r amplitudes that were recorded from ALS patients and HC. In short, LRP-r amplitudes were unaffected by Group, either alone or in interaction with CE and the CSE by Response Sequence interaction (all $F<1.10$, all $p>0.05)$. LRP-r amplitudes were enhanced on response alternation $(-3.46 \mu \mathrm{V})$ compared to response repetition trials $(-3.20 \mu \mathrm{V})$, giving rise to a statistically significant Response Sequence effect, $F_{(1,39)}=11.47, p=0.002, \eta_{p}^{2}=0.227$.

\section{Relationships with Executive Functions ALS Subgroup Comparisons}

Comparisons between HC, ALSef+, and ALSef- patients did not reveal statistically significant effects of Group on RT, ER, N2 amplitudes and s-LRP amplitudes (all $F<2.74$, all $p>0.05$ ). Note that we did not compare s-LRP onset latencies because equal sample sizes are required for jackknifing (Ulrich and Miller, 2001), and the HC group comprised 20 individuals, whereas the ALSef + and ALSef- patient groups each comprised 10 individuals.

With regard to posterior negativity amplitudes (see Figure 4), the comparison between HC, ALSef + , and ALSef- patients yielded a statistically significant Group effect, $F_{(2,37)}=4.59, p=$ $0.017, \eta_{p}^{2}=0.199$. Post-hoc tests revealed that posterior negativity amplitudes were enhanced in the ALSef- group $(-4.44 \mu \mathrm{V})$ in
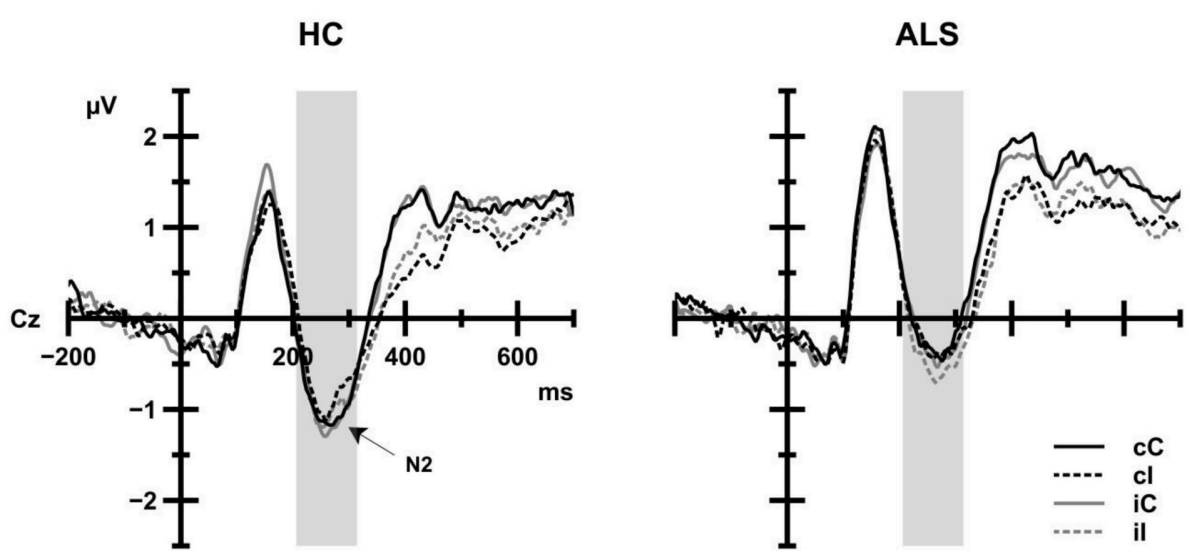

FIGURE 5 | Grand average ERP activity of HC (left panel) and ALS patients (right panel) as a function of current trial congruency (solid lines: congruent trials, dashed lines: incongruent trials) at $\mathbf{C z}$ electrode. Black lines indicate that the previous trial was congruent; gray lines indicate that the previous trial was incongruent. The shaded area indicates the time window for analyses. No group differences were observed. 
HC

ALS

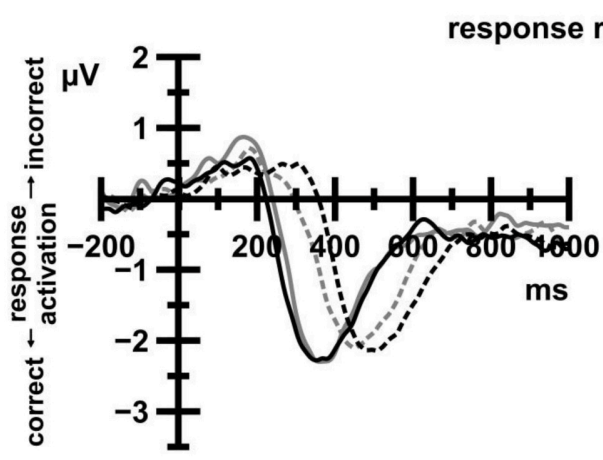

response alternation trials
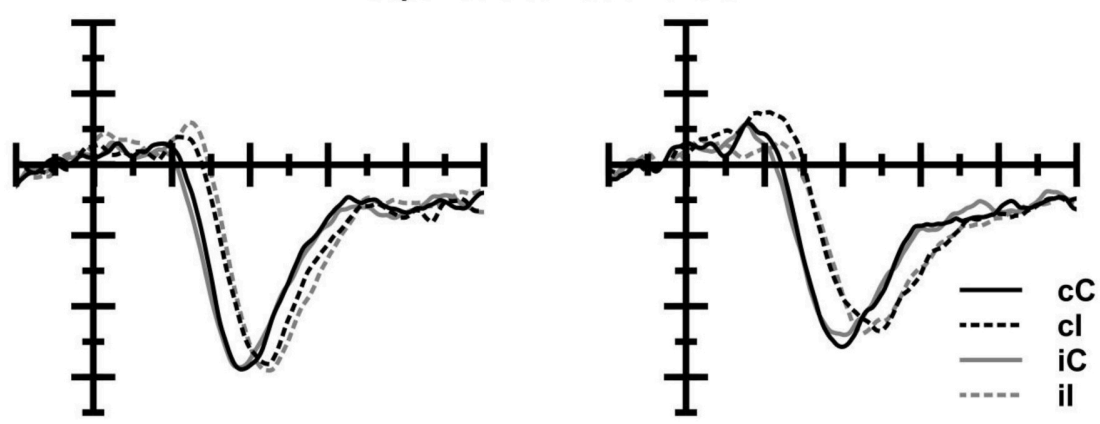

FIGURE 6 | Stimulus-locked LRP waveforms for HC (left panels) and ALS patients (right panels) as a function of current trial congruency (solid lines: congruent trials, dashed lines: incongruent trials). Black lines indicate that the previous trial was congruent; gray lines indicate that the previous trial was incongruent. Positive deflections reflect incorrect response activation; negative deflections reflect correct response activation. Response repetition trials (upper panels) exhibit a previous congruency by current congruency interaction that is absent in response alternation trials (lower panels). There were no differences between ALS patients and $\mathrm{HC}$.
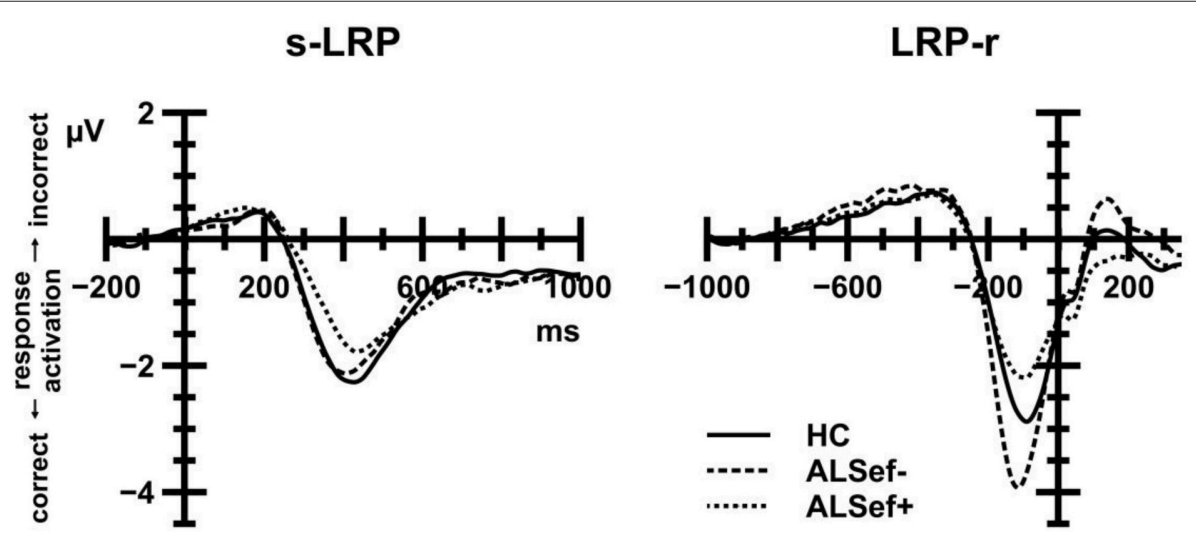

FIGURE 7 | Stimulus-locked (left panel) and response-locked (right panel) LRP waveforms. Positive deflections reflect incorrect response activation; negative deflections reflect correct response activation. To illustrate the relation between M-WCST performance and LRP amplitudes, ALS patient data were subdivided: dashed lines show LRP data for ALS patients with below-median performance on the M-WCST (ALSef-); dotted lines show LRP data for ALS patients with above-median performance on the M-WCST (ALSef+). These patient subgroups differed markedly with regard to their LRP-r amplitude preceding the overt response.

comparison to $\mathrm{HC}(-1.43 \mu \mathrm{V}), p=0.020$, while the remaining group comparisons revealed statistically non-significant results [HC vs. ALSef $+(-3.36 \mu \mathrm{V}): p=0.216$, ALSef - vs. ALSef+: $p>0.999]$.
With regard to LRP-r amplitudes (see Figure 7, right panel), the comparison between HC, ALSef+, and ALSef- patients yielded a statistically significant Group effect, $F_{(2,37)}=3.54$, $p=0.039, \eta_{p}^{2}=0.160$. Post-hoc tests revealed that LRP-r 
TABLE 2 | Correlations of executive performance in neuropsychological assessments, and behavioral performance and electrophysiological measures obtained on the flanker task for ALS patients (above diagonal, shaded) and HC (below diagonal).

\begin{tabular}{|c|c|c|c|c|c|c|c|c|}
\hline $\begin{array}{l}\text { ALS (above diagonal)/ } \\
\text { HC (below diagonal) }\end{array}$ & $\begin{array}{l}\text { M-WCST } \\
\text { EFC }\end{array}$ & ECAS EFS & RT & ER & $\begin{array}{c}\text { Posterior negativity } \\
\text { amplitude }\end{array}$ & $\begin{array}{c}\mathrm{N} 2 \\
\text { amplitude }\end{array}$ & $\begin{array}{c}\text { S-LRP } \\
\text { amplitude }\end{array}$ & $\begin{array}{l}\text { LRP-r } \\
\text { amplitude }\end{array}$ \\
\hline M-WCST EFC & - & 0.384 & 0.360 & -0.230 & 0.195 & -0.003 & $0.564^{\star *}$ & $0.574^{* *}$ \\
\hline ECAS EFS & 0.140 & - & 0.203 & $-0.446^{*}$ & 0.319 & -0.319 & 0.169 & 0.199 \\
\hline $\mathrm{RT}$ & -0.384 & 0.005 & - & -0.194 & 0.066 & 0.009 & $0.573^{\star \star}$ & $0.514^{*}$ \\
\hline ER & 0.079 & -0.118 & -0.311 & - & $-0.499^{*}$ & -0.095 & -0.231 & -0.330 \\
\hline Posterior negativity amplitude & 0.191 & -0.395 & -0.044 & -0.122 & - & -0.049 & 0.364 & $0.496^{\star}$ \\
\hline N2 amplitude & 0.348 & 0.160 & 0.162 & -0.374 & -0.233 & - & 0.174 & 0.169 \\
\hline s-LRP amplitude & -0.040 & -0.121 & 0.377 & -0.358 & 0.122 & $0.477^{\star}$ & - & $0.956^{\star \star}$ \\
\hline LRP-r amplitude & -0.017 & -0.104 & 0.359 & -0.418 & 0.111 & $0.499^{*}$ & $0.971^{\star *}$ & - \\
\hline
\end{tabular}

${ }^{\star} p<0.05,{ }^{* \star} p<0.01$.

amplitudes were enhanced in ALSef- $(-4.37 \mu \mathrm{V})$ in comparison to ALSef+ patients $(-2.61 \mu \mathrm{V}), p=0.040$, while the remaining group comparisons revealed statistically non-significant results $[\mathrm{HC}(-3.22 \mu \mathrm{V})$ vs. ALSef-: $p=0.173$; HC vs. ALSef + : $p=0.915]$.

\section{Correlation Analyses}

Since the sample size of the ALS subgroup comparisons was rather small, correlation analyses were performed in order to substantiate the reliability of the effects. Table 2 shows the results of these correlation analyses, i.e., Spearman rank correlation coefficients, separately for ALS patients (above the diagonal) and HC (below the diagonal). We chose Spearman correlations rather than Pearson correlations because they are less sensitive to strong outliers. For sake of brevity, we only comment on significant correlations between indicators of executive dysfunctions and behavioral and electrophysiological measures that were obtained on the flanker task. To begin with, ECAS EFS correlated negatively with ER $\left(r_{s}=-0.446, p=0.043\right)$ in ALS patients, indicating that better performance on the executive part of the ECAS was associated with more accurate performance. Further, LRP amplitudes (s-LRP, $r_{s}=0.564, p=0.010$; LRP-r, $r_{s}=$ $0.574, p=0.008)$ correlated with the M-WCST EFC in ALS patients, but not in $\mathrm{HC}$ (all $p>0.05$ ). It is worthy of note that these two correlation coefficients differed between ALS patients and HC (s-LRP, $z=1.98, p=0.048$; LRP-r, $z=1.95$, $p=0.051)$.

\section{DISCUSSION}

We examined 21 ALS patients and 20 age-, gender-, and education-matched control participants with a battery of neuropsychological tests and a non-verbal version of the flanker task. ALS patients showed normal performance in their response times (RT), suggesting that conflict processing and its contextual modulation are unaffected by the disease. This conclusion that can be drawn from the behavioral data was further corroborated by the ERP and LRP data in that no evidence for altered neural indices of conflict processing and its contextual modulation could be discerned. More specifically, neither N2 amplitudes nor s-LRP onset latencies were altered in ALS patients compared to HC. However, we made three more subtle, nonetheless potentially important, observations. First, ALS patients showed enhanced posterior negativity amplitudes at occipital electrodes, and this ERP enhancement was somewhat more pronounced in those patients who showed clinical evidence for some degree of executive dysfunctions (EDF) as assessed by the M-WCST EFC. Second, subgroup analyses and correlation analyses converged in the finding that the same subgroup of ALS patients showed enhanced LRP amplitudes in comparison to ALS patients without clinical signs of EDF. The LRP amplitude data suggest that the presence of EDF in ALS might be associated with functional alterations in motor regions of the cerebral cortex. Third, the presence of EDF in ALS as assessed by the ECAS EFS was associated with more error-prone behavior (ER) on the flanker task, putatively mediated through functional alterations in prefrontal regions of the cerebral cortex (Luks et al., 2010).

\section{Theoretical Integration of the Findings}

Figure 8 presents a post-hoc synthesis of these data. Note that due to the exploratory character of our study, this synthesis can only represent a preliminary interpretation of the results discussed here. Further studies are required to confirm or disconfirm these findings, and to finally understand the nature of altered information processing in ALS and the cortical correlates thereof. Here, we consider the ALS-related prefrontal TDP-43 proteinopathy as the final (i.e., neurobiological) common pathway (Arai et al., 2006; Neumann et al., 2006; Geser et al., 2008). We assume that individual differences in ALS-related prefrontal proteinopathy are associated with individual differences in clinically manifest EDF, as assessed by the M-WCST EFC and the ECAS EFS. Our non-verbal flanker study was conducted to analyze how this prefrontal proteinopathy affects information processing from perception to action, particularly conflict processing and its contextual modulation. As can be seen, behavioral measures (RT, ER) do not allow decomposing information processing into its constituent parts because altered perception, response selection and motor preparation might induce, alone or in dynamic 


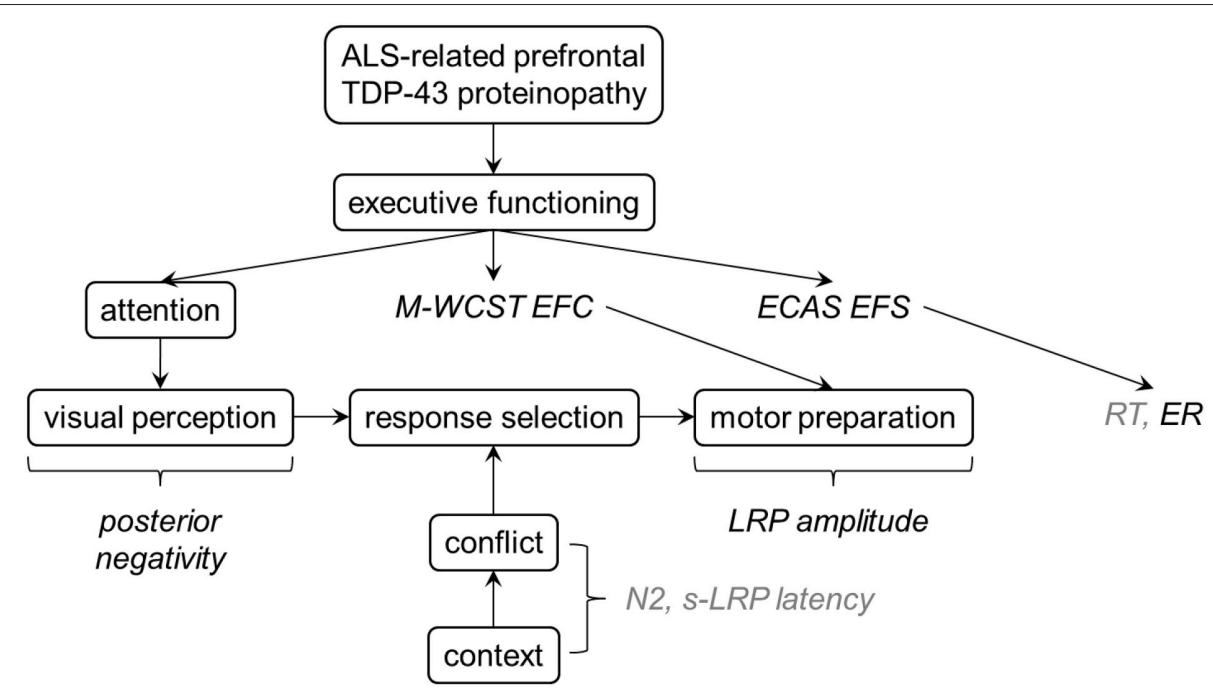

FIGURE 8 | A suggestion for post-hoc theoretical interpretation of the data. ALS-related prefrontal TDP-43 proteinopathy should be associated with measurable interindividual differences in executive functions. While response selection seems to be relatively unaffected by ALS (as revealed by normal RT, N2 amplitudes and s-LRP), enhanced posterior negativities in ALS patients might reflect functional compensation of executive deficits by stronger modulation of visual processing. LRP amplitudes were associated with EDF in ALS patients, pointing to a potential link between functional dysregulation in prefrontal and motor areas of the cortex. More error-prone behavior on the flanker task was also related to EDF in ALS patients.

interplay, alterations in RT or ER. The desired decomposition can, however, be achieved by measuring ERP and LRP because they provide relatively specific measures of neural substrates of these constituent parts. Specifically, posterior negativities reflect attentional modulation of (visual) perception, N2 amplitudes and s-LRP onset latencies are sensitive to conflict processing and its contextual modulation, and LRP amplitudes offer insights into cortical mechanisms of motor preparation. Our findings suggest four major conclusions: First, response selection seems generally unaffected by the disease. In particular, conflict processing and its contextual modulation are spared by the ALS-related prefrontal proteinopathy, as revealed by the unaltered response times (RT), N2 amplitudes and s-LRP onset latencies in ALS patients (Figure 8, italics in light gray). Second, declines in executive abilities appear to be functionally compensated by increased modulation of visual processing by frontoparietal networks in ALS, as revealed by enhanced posterior negativities (Figure 8, italics in black). Third, individual differences in clinically manifest EDF, as assessed by the M-WCST EFC, are associated with enhanced LRP amplitudes in ALS patients, pointing to a potential link between functional dysregulation in prefrontal and motor areas of the cerebral cortex in ALS. Fourth, individual differences in clinically manifest EDF, as assessed by the ECAS EFS, are associated with error-prone behavior (ER) on the flanker task, pointing to prefrontal functional dysregulation (Luks et al., 2010). For sake of brevity, we will only shortly comment on each of these conclusions in the remainder of this discussion.

\section{Conflict Processing in ALS Patients}

The ability to efficiently select motor responses appears to be unaffected by ALS, both under non-conflicting as well as under conflicting conditions. Further, we could not discern evidence (behavioral, neural) for ALS-related alterations with regard to the well-documented (see Section Introduction) contextual modulation of conflict processing. Of course, null findings should be treated with caution; however, they stand in contrast to earlier findings from our group that we obtained from PD patients (Rustamov et al., 2013). In this flanker study, we found that PD is associated with alterations in the contextual modulation of conflict processing. Further research is required to examine directly potential dissociations between ALS and PD with regard to the contextual modulation of conflict processing.

\section{Attentional Modulation of Visual Perception in ALS Patients}

As outlined in the Introduction, posterior negativities are subject to prefrontal modulation (Barceló et al., 2000). Following the evidence from that study, modulations of posterior negativities have their origin on frontoparietal attention networks (Ptak, 2012; Vossel et al., 2014). According to this approach, attentional control involves the setting of task-driven priorities to bias competition in visuospatial feature maps thus affecting the information gain from task-relevant and task-irrelevant parts of a visual scene. Attentional control as it applies to flanker tasks is discussed in detail in Rustamov et al. (2014).

ALS patients, in particular those with clinically detectable EDF, showed enhanced posterior negativity amplitudes in comparison to HC. Given evidence for prefrontal control over posterior negativity amplitudes, these data might indicate a taskdriven "sharpening" of visual processing (Rustamov et al., 2014), putatively in the service of functional compensation (ReuterLorenz and Cappell, 2008) and probably through additional mechanisms recruited in frontoparietal attention networks (Ptak, 2012; Vossel et al., 2014). If so, the present posterior negativity 
finding is in line with multiple neuroimaging studies that used various paradigms, and that demonstrated a diversity of patterns of enhanced cortical activity in ALS patients (Kew et al., 1993b; Schoenfeld et al., 2005; Han and Ma, 2006; Stanton et al., 2006; Lulé et al., 2007; Dounaud et al., 2011; Goldstein et al., 2011; Mohammadi et al., 2011, 2015; Cosottini et al., 2012; Witiuk et al., 2014; see Turner et al., 2012, for review).

\section{Motor Preparation in ALS Patients}

The LRP is a quantification of the inequality of the ERPs contralateral and ipsilateral to the hand making the response. The two competing hands, left vs. right, are in equal activation until one side gets the advantage. When the disparity between the two hands becomes non-zero, the LRP has its onset. LRP amplitudes are of considerable interest in the light of a number of studies that showed that LRP amplitudes are enhanced in elderly participants in comparison to young participants (Yordanova et al., 2004; Roggeveen et al., 2007; Wild-Wall et al., 2008; Vallesi and Stuss, 2010; Cespón et al., 2013; Cid-Fernández et al., 2014). According to one hypothesis, increased LRP amplitudes in older adults arise from reduced inhibition (mediated by GABAergic synapses) within the motor cortex (Roggeveen et al., 2007). When inhibition is weaker, both activations ultimately build up to higher levels, and enhanced LRP amplitudes will result to the extent of stronger disinhibition for the responding hand than for the competing hand. Alternatively, enhanced LRP amplitudes were associated with dysregulation in high-level control networks on the basis of the compensation hypothesis (Reuter-Lorenz and Cappell, 2008), suggesting that enhanced LRP amplitudes may be related to additional mechanisms recruited for maintaining motor performance (Wild-Wall et al., 2008).

In contrast to normal aging, ALS itself does not seem to be associated with enhanced LRP amplitudes. However, subgroup analyses and correlation analyses showed that clinically detectable ALS-related EDF (as assessed by the M-WCST EFC) are related to enhanced LRP amplitudes. The specificity of the relationship between EDF and enhanced LRP amplitudes suggests that the LRP amplitude enhancement in ALS patients with EDF might occur as a corollary of the dysregulation in prefrontal control networks that in turn may be related to additional mechanisms recruited for maintaining motor performance.

\section{Error-Proneness in ALS Patients}

The ECAS (Abrahams et al., 2014) allowed examining aspects of executive functions beyond those assessed by the M-WCST. Performance deficits on the ECAS EFS predicted higher ER on the flanker task. Luks et al. (2010) found that atrophy of the left hemisphere dIPFC and ACC in 65 patients with various neurodegenerative diseases (frontotemporal lobar degeneration, Alzheimer's disease, corticobasal degeneration, or progressive supranuclear palsy, but not ALS) predicted higher ER on the flanker task. It is thus possible that the relationship between ECAS EFS and ER might be mediated by dysfunction of the left hemisphere dIPFC and ACC. The proposed relationship remains to be elucidated in future studies using structural and functional imaging techniques.

\section{Study Limitations}

Replication in independent, larger samples of ALS patients is warranted, in particular to ensure the validity of the subgroup comparisons. Future studies should also consider the multidimensional nature of executive functions (Miyake et al., 2000). Finally, we suggest comparing ALS patients with patients suffering from other neurodegenerative diseases in future studies. The accomplishment of a comparative approach would allow investigating the degree of specificity of cognitive and behavioral disturbances that are associated with ALS, ultimately leading to a better understanding of the neural underpinnings of these psychological disturbances.

\section{CONCLUSIONS}

This article demonstrates the utility of ERP and LRP measures that provide the means for decomposing psychological disturbances associated with ALS into constituent parts (Figure 8). Here, we focused on executive functioning which is important for the ability to predict behavioral sequelae and course of the disease (Olney et al., 2005; Chiò et al., 2010; Elamin et al., 2011; Lillo et al., 2012; Montuschi et al., 2015). Our results exemplify how electrophysiological measures might contribute to a better clinical assessment as well as to a more rigorous scientific investigation of cognitive dysfunctions in ALS patients (see also Raggi et al., 2010; Lange et al., 2015).

Accumulating evidence about the heterogeneous nature of ALS raised the awareness about its presumably multifaceted etiology and phenomenology. Recently, this has led to the hypothesis of ALS being a general term for a variety of related yet distinct disorders, rather than a clearly defined disease (Goldstein and Abrahams, 2013; Turner et al., 2013). Our results thus provide support for the need of studies disentangling these ALS subtypes in order to adequately characterize the individual problems associated with the disease and to find the optimal treatment for individual patients. Our data highlight the promising role of the ERP technique as a tool to overcome difficulties associated with purely behavioral examination techniques (Goldstein and Abrahams, 2013), and they support the previously suggested distinction of ALS with and without cognitive involvement (Ringholz et al., 2005; Lillo and Hodges, 2010; Phukan et al., 2012).

\section{AUTHOR CONTRIBUTIONS}

Conceived the study: RD, SP, BK. Designed the experiment: CS, SF, SP, BK. Performed the experiment: CS, SF, MV, SA. Analyzed the data: CS, SF, MV, FL, SA, BK. Wrote the manuscript: CS, SF, $\mathrm{MV}, \mathrm{FL}, \mathrm{RD}, \mathrm{SP}, \mathrm{BK}$. All authors provided critical revisions to the manuscript and approved the final version of the paper.

\section{ACKNOWLEDGMENTS}

This work was supported by a research grant to BK (PetermaxMüller-Stiftung, Hannover, Germany) and by the German Network for Motor Neuron Diseases (BMBF 01GM1103A) (SP, $\mathrm{RD})$. FL received a grant from the German National Academic Foundation. 


\section{REFERENCES}

Abdulla, S., Vielhaber, S., Körner, S., Machts, J., Heinze, H. J., Dengler, R., et al. (2013). Validation of the German version of the extended ALS functional rating scale as a patient-reported outcome measure. J. Neurol. 260, 2242-2255. doi: 10.1007/s00415-013-6955-6

Abe, K., Fujimura, H., Toyooka, K., Sakoda, S., Yorifuji, S., and Yanagihara, T. (1997). Cognitive function in amyotrophic lateral sclerosis. J. Neurol. Sci. 148, 95-100. doi: 10.1016/S0022-510X(96)05338-5

Abrahams, S. (2011). Social cognition in amyotrophic lateral sclerosis. Neurodegener. Dis. Manag. 1, 397-405. doi: 10.2217/nmt.11.54

Abrahams, S. (2013). Executive dysfunction is not the whole story. J. Neurol. Neurosurg. Psychiatry 84, 474-475. doi: 10.1136/jnnp-2012-303851

Abrahams, S., Goldstein, L. H., Al-Chalabi, A., Pickering, A., Morris, R. G., Passingham, R. E., et al. (1997). Relation between cognitive dysfunction and pseudobulbar palsy in amyotrophic lateral sclerosis. J. Neurol. Neurosurg. Psychiatry 62, 464-472. doi: 10.1136/jnnp.62.5.464

Abrahams, S., Goldstein, L. H., Kew, J. J. M., Brooks, D. J., Lloyd, C. M., Frith, C. D., et al. (1996). Frontal lobe dysfunction in amyotrophic lateral sclerosis: a PET study. Brain 119, 2105-2120. doi: 10.1093/brain/119.6.2105

Abrahams, S., Goldstein, L. H., Simmons, A., Brammer, M., Williams, S. C. R., Giampietro, V., et al. (2004). Word retrieval in amyotrophic lateral sclerosis: a functional magnetic resonance imaging study. Brain 127, 1507-1517. doi: 10.1093/brain/awh170

Abrahams, S., Goldstein, L. H., Suckling, J., Ng, V., Simmons, A., Chitnis, X., et al. (2005a). Frontotemporal white matter changes in amyotrophic lateral sclerosis. J. Neurol. 252, 321-331. doi: 10.1007/s00415-005-0646-x

Abrahams, S., Leigh, P. N., and Goldstein, L. H. (2005b). Cognitive change in ALS: a prospective study. Neurology 64, 1222-1226. doi: 10.1212/01.WNL.0000156519.41681.27

Abrahams, S., Leigh, P. N., Harvey, A., Vythelingum, G. N., Grise, D., and Goldstein, L. H. (2000). Verbal fluency and executive dysfunction in amyotrophic lateral sclerosis (ALS). Neuropsychologia 38, 734-747. doi: 10.1016/S0028-3932(99)00146-3

Abrahams, S., Newton, J., Niven, E., Foley, J., and Bak, T. H. (2014). Screening for cognition and behaviour changes in ALS. Amyotroph. Lateral Scler. Frontotemporal Degener. 15, 9-14. doi: 10.3109/21678421.2013.805784

Agosta, F., Canu, E., Valsasina, P., Riva, N., Prelle, A., Comi, G., et al. (2013). Divergent brain network connectivity in amyotrophic lateral sclerosis. Neurobiol. Aging 34, 419-427. doi: 10.1016/j.neurobiolaging.2012.04.015

Al-Chalabi, A., Jones, A., Troakes, C., King, A., Al-Sarraj, S., and van den Berg, L. H. (2012). The genetics and neuropathology of amyotrophic lateral sclerosis. Acta Neuropathol. 124, 339-352. doi: 10.1007/s00401-012-1022-4

Amato, N., Riva, N., Cursi, M., Martins-Silva, A., Martinelli, V., Comola, M., et al. (2013). Different frontal involvement in ALS and PLS revealed by Stroop event-related potentials and reaction times. Front. Aging Neurosci. 5:82. doi: 10.3389/fnagi.2013.00082

Arai, T., Hasegawa, M., Akiyama, H., Ikeda, K., Nonaka, T., Mori, H., et al. (2006). TDP-43 is a component of ubiquitin-positive tau-negative inclusions in frontotemporal lobar degeneration and amyotrophic lateral sclerosis. Biochem. Biophys. Res. Commun. 351, 602-611. doi: 10.1016/j.bbrc.2006.10.093

Barceló, F., Suwazono, S., and Knight, R. T. (2000). Prefrontal modulation of visual processing in humans. Nat. Neurosci. 3, 399-403. doi: 10.1038/73975

Botvinick, M. M., Braver, T. S., Barch, D. M., Carter, C. S., and Cohen, J. D. (2001). Conflict monitoring and cognitive control. Psychol. Rev. 108, 624-652. doi: 10.1037/0033-295X.108.3.624

Brooks, B. R., Miller, R. G., Swash, M., and Munsat, T. L. (2000). El Escorial revisited: revised criteria for the diagnosis of amyotrophic lateral sclerosis. Amyotroph. Lateral Scler. 1, 293-299. doi: 10.1080/146608200300079536

Cedarbaum, J. M., Stambler, N., Malta, E., Fuller, C., Hilt, D., Thurmond, B., et al. (1999). The ALSFRS-R: a revised ALS functional rating scale that incorporates assessments of respiratory function. J. Neurol. Sci. 169, 13-21. doi: 10.1016/S0022-510X(99)00210-5

Cespón, J., Galdo-Álvarez, S., and Díaz, F. (2013). Age-related changes in ERP correlates of visuospatial and motor processes. Psychophysiology 50, 743-757. doi: 10.1111 psyp. 12063

Chari, G., Shaw, P. J., and Sahgal, A. (1996). Nonverbal visual attention, but not recognition memory or learning, processes are impaired in motor neurone disease. Neuropsychologia 34, 377-385. doi: 10.1016/0028-3932(95) 00122-0

Chiò, A., Vignola, A., Mastro, E., Giudici, A. D., Iazzolino, B., Calvo, A., et al. (2010). Neurobehavioral symptoms in ALS are negatively related to caregivers' burden and quality of life. Eur. J. Neurol. 17, 1298-1303. doi: 10.1111/j.14681331.2010.03016.x

Christidi, F., Zalonis, I., Smyrnis, N., and Evdokimidis, I. (2012). Selective attention and the three-process memory model for the interpretation of verbal free recall in amyotrophic lateral sclerosis. J. Int. Neuropsych. Soc. 18, 809-818. doi: 10.1017/S1355617712000562

Cid-Fernández, S., Lindín, M., and Díaz, F. (2014). Effects of aging and involuntary capture of attention on event-related potentials associated with the processing of and the response to a target stimulus. Front. Hum. Neurosci. 8:745. doi: 10.3389/fnhum.2014.00745

Clayson, P. E., and Larson, M. J. (2011a). Conflict adaptation and sequential trial effects: support for the conflict monitoring theory. Neuropsychologia 49, 1953-1961. doi: 10.1016/j.neuropsychologia.2011.03.023

Clayson, P. E., and Larson, M. J. (2011b). Effects of repetition priming on electrophysiological and behavioral indices of conflict adaptation and cognitive control. Psychophysiology 48, 1621-1630. doi: 10.1111/j.14698986.2011.01265.x

Clayson, P. E., and Larson, M. J. (2012). Cognitive performance and electrophysiological indices of cognitive control: a validation study of conflict adaptation. Psychophysiology 49, 627-637. doi: 10.1111/j.14698986.2011.01345.x

Coles, M. G. (1989). Modern mind-brain reading: psychophysiology, physiology, and cognition. Psychophysiology 26, 251-269. doi: 10.1111/j.1469-8986.1989.tb01916.x

Cosottini, M., Pesaresi, I., Piazza, S., Diciotti, S., Cecchi, P., Fabbri, S., et al. (2012). Structural and functional evaluation of cortical motor areas in amyotrophic lateral sclerosis. Exp. Neurol. 234, 169-180. doi: 10.1016/j.expneurol.2011.12.024

Danielmeier, C., Wessel, J. R., Steinhauser, M., and Ullsperger, M. (2009). Modulation of the error-related negativity by response conflict. Psychophysiology 46, 1288-1298. doi: 10.1111/j.1469-8986.2009.00860.x

DeJesus-Hernandez, M., Mackenzie, I. R., Boeve, B. F., Boxer, A. L., Baker, M., Rutherford, N. J., et al. (2011). Expanded GGGGCC hexanucleotide repeat in noncoding region of C9ORF72 causes chromosome 9p-linked FTD and ALS. Neuron 72, 245-256. doi: 10.1016/j.neuron.2011.09.011

Dounaud, G., Filippini, N., Knight, S., Talbot, K., and Turner, M. R. (2011). Integration of structural and functional magnetic resonance imaging in amyotrophic lateral sclerosis. Brain 134, 3470-3479. doi: 10.1093/brain/awr279

Dubois, B., Slachevsky, A., Litvan, I., and Pillon, B. (2000). The FAB: a frontal assessment battery at bedside. Neurology 55, 1621-1626. doi: 10.1212/WNL.55.11.1621

Eder, A. B., Leuthold, H., Rothermund, K., and Schweinberger, S. R. (2012). Automatic response activation in sequential affective priming: an ERP study. Soc. Cogn. Affect. Neurosci. 7, 436-445. doi: 10.1093/scan/nsr033

Egner, T. (2007). Congruency sequence effects and cognitive control. Cogn. Affect. Behav. Neurosci. 7, 380-390. doi: 10.3758/CABN.7.4.380

Eimer, M. (1998). The lateralized readiness potential as an on-line measure of central response activation processes. Behav. Res. Methods Instrum. Comput. 30, 146-156. doi: 10.3758/BF03209424

Elamin, M., Phukan, J., Bede, P., Jordan, N., Byrne, S., Pender, N., et al. (2011). Executive dysfunction is a negative prognostic indicator in patients with ALS without dementia. Neurology 76, 1263-1269. doi: 10.1212/WNL.0b013e318214359f

Eriksen, B. A., and Eriksen, C. W. (1974). Effects of noise letters upon the identification of a target letter in a nonsearch task. Percep. Psychophys. 16, 143-149. doi: 10.3758/BF03203267

Folstein, J. R., and Van Petten, C. (2008). Influence of cognitive control and mismatch on the N2 component of the ERP: a review. Psychophysiology 45, 152-170. doi: 10.1111/j.1469-8986.2007.00602.x

Forster, S. E., Carter, C. S., Cohen, J. D., and Cho, R. Y. (2011). Parametric manipulation of the conflict signal and control-state adaptation. J. Cogn. Neurosci. 23, 923-935. doi: 10.1162/jocn.2010.21458

Frank, B., Haas, J., Heinze, H. J., Stark, E., and Münte, T. F. (1997). Relation of neuropsychological and magnetic resonance findings in amyotrophic lateral 
sclerosis: evidence for subgroups. Clin. Neurol. Neurosurg. 99, 79-86. doi: 10.1016/S0303-8467(97)80001-7

Freitas, A. L., Banai, R., and Clark, S. L. (2009). When cognitive control is calibrated: event-related potential correlates of adapting to information-processing conflict despite erroneous response preparation. Psychophysiology 46, 1226-1233. doi: 10.1111/j.1469-8986.2009.00864.x

Geser, F., Brandmeir, N. J., Kwong, L. K., Martinez-Lage, M., Elman, L., McCluskey, L., et al. (2008). Evidence of multisystem disorder in whole-brain map of pathological TDP-43 in amyotrophic lateral sclerosis. Arch. Neurol. 65, 636-641. doi: 10.1001/archneur.65.5.636

Gibbons, Z. C., Richardson, A., Neary, D., and Snowden, J. S. (2008). Behaviour in amyotrophic lateral sclerosis. Amyotroph. Lateral Scler. 9, 67-74. doi: $10.1080 / 17482960701642437$

Gil, R., Neau, J. P., Dary-Auriol, M., Agbo, C., Tantot, A. M., and Ingrand, P. (1995). Event-related auditory evoked potentials and amyotrophic lateral sclerosis. Arch. Neurol. 52, 890. doi: 10.1001/archneur.1995.00540330068017

Goldstein, L. H., and Abrahams, S. (2013). Changes in cognition and behaviour in amyotrophic lateral sclerosis: nature of impairment and implications for assessment. Lancet Neurol. 12, 368-380. doi: 10.1016/S1474-4422(13)70026-7

Goldstein, L. H., Newsom-Davis, I. C., Bryant, V., Brammer, M., Leigh, P. N., and Simmons, A. (2011). Altered patterns of cortical activation in ALS patients during attention and cognitive response inhibition tasks. J. Neurol. 258, 2186-2198. doi: 10.1007/s00415-011-6088-8

Gratton, G., Coles, M. G., and Donchin, E. (1992). Optimizing the use of information: strategic control of activation of responses. J. Exp. Psychol. Gen. 121, 480-506. doi: 10.1037/0096-3445.121.4.480

Gratton, G., Coles, M. G., Sirevaag, E. J., Eriksen, C. W., and Donchin, E. (1988). Pre- and poststimulus activation of response channels: a psychophysiological analysis. J. Exp. Psychol. Hum. Percept. Perform. 14, 331-344. doi: 10.1037/0096-1523.14.3.331

Groppe, D. M., Makeig, S., and Kutas, M. (2008). Independent component analysis of event-related potentials. Cogn. Sci. Online 6, 1-44.

Grossman, A. B., Woolley-Levine, S., Bradley, W. G., and Miller, R. G. (2007). Detecting neurobehavioral changes in amyotrophic lateral sclerosis. Amyotroph. Lateral Scler. 8, 56-61. doi: 10.1080/17482960601044106

Han, J., and Ma, L. (2006). Functional magnetic resonance imaging study of the brain in patients with amyotrophic lateral sclerosis. Chin. Med. Sci. J. 21, 228-233.

Hanagasi, H. A., Gurvit, I. H., Ermutlu, N., Kaptanoglu, G., Karamursel, S., Idrisoglu, H. A., et al. (2002). Cognitive impairment in amyotrophic lateral sclerosis: evidence from neuropsychological investigation and event-related potentials. Cogn. Brain Res. 14, 234-244. doi: 10.1016/S0926-6410(02)00110-6

Heaton, R. K., Chelune, G. J., Talley, J. L., Kay, G. G., and Curtiss, G. (1993). Wisconsin Card Sorting Test Manual: Revised and Expanded. Odessa, FL: Psychological Assessment Resources.

Hillyard, S. A., and Anllo-Vento, L. (1998). Event-related brain potentials in the study of visual selective attention. Proc. Natl. Acad. Sci. U.S.A. 95, 781-787.

Hillyard, S. A., Teder-Sälejärvi, W. A., and Münte, T. F. (1998). Temporal dynamics of early perceptual processing. Curr. Opin. Neurobiol. 8, 202-210. doi: 10.1016/S0959-4388(98)80141-4

Johns, M. W. (1991). A new method for measuring daytime sleepiness: the Epworth sleepiness scale. Sleep 14, 540-545.

Kew, J. J. M., Goldstein, L. H., Leigh, P. N., Abrahams, S., Cosgrave, N., Passingham, R. E., et al. (1993a). The relationship between abnormalities of cognitive function and cerebral activation in amyotrophic lateral sclerosis: a neuropsychological and positron emission tomography study. Brain 116, 1399-1423. doi: 10.1093/brain/116.6.1399

Kew, J. J. M., Leigh, P. N., Playford, E. D., Passingham, R. E., Goldstein, L. H., Frackowiak, R. S. J., et al. (1993b). Cortical function in amyotrophic lateral sclerosis: a positron emission tomography study. Brain 116, 655-680. doi: 10.1093/brain/116.3.655

Kiernan, M. C., Vucic, S., Cheah, B. C., Turner, M. R., Eisen, A., Hardiman, O., et al. (2011). Amyotrophic lateral sclerosis. Lancet 377, 942-955. doi: 10.1016/S0140-6736(10)61156-7

Kiesel, A., Miller, J., Jolicœur, P., and Brisson, B. (2008). Measurement of ERP latency differences: a comparison of single-participant and jackknife-based scoring methods. Psychophysiology 45, 250-274. doi: 10.1111/j.1469-8986.2007.00618.x
Kopp, B., Rist, F., and Mattler, U. (1996). N200 in the flanker task as a neurobehavioral tool for investigating executive control. Psychophysiology 33, 282-294. doi: 10.1111/j.1469-8986.1996.tb00425.x

Kopp, B., Rösser, N., Tabeling, S., Stürenburg, H. J., de Haan, B., Karnath, H. O., et al. (2013). Performance on the frontal assessment battery is sensitive to frontal lobe damage in stroke patients. BMC Neurol. 13:179. doi: 10.1186/14712377-13-179

Kopp, B., Tabeling, S., Moschner, C., and Wessel, K. (2007). Temporal dynamics of selective attention and conflict resolution during cross-dimensional go-nogo decisions. BMC Neurosci. 8:68. doi: 10.1186/1471-2202-8-68

Kopp, B., and Wessel, K. (2010). Event-related brain potentials and cognitive processes related to perceptual-motor information transmission. Cogn. Affect. Behav. Neurosci. 10, 316-327. doi: 10.3758/CABN.10.2.316

Lange, F., Vogts, M.-B., Seer, C., Fürkötter, S., Abdulla, S., Dengler, R., et al. (2015). Impaired set-shifting in amyotrophic lateral sclerosis: an event-related potential study of executive function. Neuropsychology. doi: 10.1037/neu0000218. [Epub ahead of print].

Larson, M. J., Clayson, P. E., and Baldwin, S. A. (2012). Performance monitoring following conflict: internal adjustments in cognitive control? Neuropsychologia 50, 426-433 doi: 10.1016/j.neuropsychologia.2011.12.021

Leuthold, H., and Jentzsch, I. (2002). Distinguishing neural sources of movement preparation and execution: an electrophysiological analysis. Biol. Psychol. 60, 173-198. doi: 10.1016/S0301-0511(02)00032-7

Lillo, P., and Hodges, J. R. (2010). Cognition and behaviour in motor neurone disease. Curr. Opin. Neurol. 23, 638-642. doi: 10.1097/WCO.0b013e3283400b41

Lillo, P., Mioshi, E., Zoing, M. C., Kiernan, M. C., and Hodges, J. R. (2011). How common are behavioural changes in amyotrophic lateral sclerosis? Amyotroph. Lateral Scler. 12, 45-51. doi: 10.3109/17482968.2010.520718

Lillo, P., Savage, S., Mioshi, E., Kiernan, M. C., and Hodges, J. R. (2012). Amyotrophic lateral sclerosis and frontotemporal dementia: a behavioural and cognitive continuum. Amyotroph. Lateral Scler. 13, 102-109. doi: $10.3109 / 17482968.2011 .639376$

Logroscino, G., Traynor, B. J., Hardiman, O., Chiò, A., Mitchell, D., Swingler, R. J., et al. (2010). Incidence of amyotrophic lateral sclerosis in Europe. J. Neurol. Neurosurg. Psychiatry 81, 385-390. doi: 10.1136/jnnp.2009.183525

Lomen-Hoerth, C., Murphy, J., Langmore, S., Kramer, J. H., Olney, R. K., and Miller, B. (2003). Are amyotrophic lateral sclerosis patients cognitively normal? Neurology 60, 1094-1097. doi: 10.1212/01.WNL.0000055861.95202.8D

Luck, S. J. (2005). An Introduction to the Event-related Potential Technique. Cambridge, MA: MIT Press.

Ludolph, A. C., Langen, K. J., Regard, M., Herzog, H., Kemper, B., Kuwert, T., et al. (1992). Frontal lobe function in amyotrophic lateral sclerosis: a neuropsychologic and positron emission tomography study. Acta Neurol. Scand. 85, 81-89. doi: 10.1111/j.1600-0404.1992. tb04003.x

Luks, T. L., Oliveira, M., Possin, K. L., Bird, A., Miller, B. L., Weiner, M. W., et al. (2010). Atrophy in two attention networks is associated with performance on a Flanker task in neurodegenerative disease. Neuropsychologia 48, 165-170. doi: 10.1016/j.neuropsychologia.2009.09.001

Lulé, D., Diekmann, V., Kassubek, J., Kurt, A., Birbaumer, N., Ludolph, A. C., et al. (2007). Cortical plasticity in amyotrophic lateral sclerosis: motor imagery and function. Neurorehabil. Neural. Repair 21, 518-526. doi: $10.1177 / 1545968307300698$

Massman, P. J., Sims, J., Cooke, N., Haverkamp, L. J., Appel, V., and Appel, S. H. (1996). Prevalence and correlates of neuropsychological deficits in amyotrophic lateral sclerosis. J. Neurol. Neurosurg. Psychiatry 61, 450-455. doi: 10.1136/jnnp.61.5.450

Mayr, U., Awh, E., and Laurey, P. (2003). Conflict adaptation effects in the absence of executive control. Nat. Neurosci. 6, 450-452. doi: 10.1038/nn1051

Miller, J., Patterson, T., and Ulrich, R. (1998). Jackknife-based method for measuring LRP onset latency differences. Psychophysiology 35, 99-115.

Miyake, A., Friedman, N. P., Emerson, M. J., Witzki, A. H., Howerter, A., and Wager, T. D. (2000). The unity and diversity of executive functions and their contributions to complex "frontal lobe" tasks: a latent variable analysis. Cogn. Psychol. 41, 49-100. doi: 10.1006/cogp.1999.0734

Mohammadi, B., Kollewe, K., Cole, D. M., Fellbrich, A., Heldmann, M., Samii, A., et al. (2015). Amyotrophic lateral sclerosis affects cortical and subcortical 
activity underlying motor inhibition and action monitoring. Hum. Brain Mapp. 36, 2878-2889. doi: 10.1002/hbm.22814

Mohammadi, B., Kollewe, K., Samii, A., Dengler, R., and Münte, T. F. (2011). Functional neuroimaging at different disease stages reveals distinct phases of neuroplastic changes in amyotrophic lateral sclerosis. Hum. Brain Mapp. 32, 750-758. doi: 10.1002/hbm.21064

Montuschi, A., Iazzolino, B., Calvo, A., Moglia, C., Lopiano, L., Restagno, G., et al. (2015). Cognitive correlates in amyotrophic lateral sclerosis: a populationbased study in Italy. J. Neurol. Neurosurg. Psychiatry 86, 168-173. doi: 10.1136/jnnp-2013-307223

Moretti, R., Torre, P., Antonello, R. M., Carraro, N., Cazzato, G., and Bava, A. (2002). Complex cognitive disruption in motor neuron disease. Dement. Geriatr. Cogn. 14, 141-150. doi: 10.1159/000063600

Münte, T. F., Tröger, M. C., Nusser, I., Wieringa, B. M., Matzke, M., Johannes, S., et al. (1999). Abnormalities of visual search behaviour in ALS patients detected with event-related brain potentials. Amyotroph. Lateral Scler. Other Motor Neuron Disord. 1, 21-27.

Nasreddine, Z. S., Phillips, N. A., Bédirian, V., Charbonneau, S., Whitehead, V., Collin, I., et al. (2005). The Montreal Cognitive Assessment, MoCA: a brief screening tool for mild cognitive impairment. J. Am. Geriatr. Soc. 53, 695-699. doi: 10.1111/j.1532-5415.2005.53221.x

Neary, D., Snowden, J. S., Gustafson, L., Passant, U., Stuss, D., Black, S. A., et al. (1998). Frontotemporal lobar degeneration: a consensus on clinical diagnostic criteria. Neurology 51, 1546-1554. doi: 10.1212/WNL.51.6.1546

Neary, D., Snowden, J. S., and Mann, D. M. A. (2000). Cognitive change in motor neurone disease/amyotrophic lateral sclerosis (MND/ALS). J. Neurol. Sci. 180, 15-20. doi: 10.1016/S0022-510X(00)00425-1

Neary, D., Snowden, J. S., Mann, D. M., Northen, B., Goulding, P. J., and Macdermott, N. (1990). Frontal lobe dementia and motor neuron disease. J. Neurol. Neurosurg. Psychiatry 53, 23-32. doi: 10.1136/jnnp.53.1.23

Neumann, M., Sampathu, D. M., Kwong, L. K., Truax, A. C., Micsenyi, M. C., Chou, T. T., et al. (2006). Ubiquitinated TDP-43 in frontotemporal lobar degeneration and amyotrophic lateral sclerosis. Science 314, 130-133. doi: $10.1126 /$ science. 1134108

Neumann, N., and Kotchoubey, B. (2004). Assessment of cognitive functions in severely paralysed and severely brain-damaged patients: neuropsychological and electrophysiological methods. Brain Res. Protoc. 14, 25-36. doi: 10.1016/j.brainresprot.2004.09.001

Olney, R. K., Murphy, J., Forshew, D. B. S. N., Garwood, E., Miller, B. L., Langmore, S., et al. (2005). The effects of executive and behavioral dysfunction on the course of ALS. Neurology 65, 1774-1777. doi: 10.1212/01.wnl.0000188759.87240.8b

Paulus, K. S., Magnano, I., Piras, M. R., Solinas, M. A., Solinas, G., Sau, G. F., et al. (2002). Visual and auditory event-related potentials in sporadic amyotrophic lateral sclerosis. Clin. Neurophysiol. 113, 853-861. doi: 10.1016/S13882457(02)00082-2

Pettit, L. D., Bastin, M. E., Smith, C., Bak, T. H., Gillingwater, T. H., and Abrahams, S. (2013). Executive deficits, not processing speed relates to abnormalities in distinct prefrontal tracts in amyotrophic lateral sclerosis. Brain 136, 3290-3304. doi: 10.1093/brain/awt243

Phukan, J., Elamin, M., Bede, P., Jordan, N., Gallagher, L., Byrne, S., et al. (2012). The syndrome of cognitive impairment in amyotrophic lateral sclerosis: a population-based study. J. Neurol. Neurosurg. Psychiatry 83, 102-108. doi: 10.1136/jnnp-2011-300188

Phukan, J., Pender, N. P., and Hardiman, O. (2007). Cognitive impairment in amyotrophic lateral sclerosis. Lancet Neurol. 6, 994-1003. doi: 10.1016/S14744422(07)70265-X

Pinkhardt, E. H., Jürgens, R., Becker, W., Mölle, M., Born, J., Ludolph, A. C., et al. (2008). Signs of impaired selective attention in patients with amyotrophic lateral sclerosis. J. Neurol. 255, 532-538. doi: 10.1007/s00415-0080734-9

Polich, J. (2007). Updating P300: an integrative theory of P3a and P3b. Clin. Neurophysiol. 118, 2128-2148. doi: 10.1016/j.clinph.2007.04.019

Ptak, R. (2012). The frontoparietal attention network of the human brain action, saliency, and a priority map of the environment. Neuroscientist 18, 502-515. doi: $10.1177 / 1073858411409051$

Raaphorst, J., de Visser, M., Linssen, W. H., de Haan, R. J., and Schmand, B. (2010). The cognitive profile of amyotrophic lateral sclerosis: a meta-analysis. Amyotroph. Lateral Scler. 11, 27-37. doi: 10.3109/17482960802645008
Raggi, A., Consonni, M., Iannaccone, S., Perani, D., Zamboni, M., Sferrazza, B., et al. (2008). Auditory event-related potentials in non-demented patients with sporadic amyotrophic lateral sclerosis. Clin. Neurophysiol. 119, 342-350. doi: 10.1016/j.clinph.2007.10.010

Raggi, A., Iannaccone, S., and Cappa, S. F. (2010). Event-related brain potentials in amyotrophic lateral sclerosis: a review of the international literature. Amyotroph. Lateral Scler. 11, 16-26. doi: 10.3109/17482960902912399

Rakowicz, W. P., and Hodges, J. R. (1998). Dementia and aphasia in motor neuron disease: an underrecognised association? J. Neurol. Neurosurg. Psychiatry 65, 881-889. doi: 10.1136/jnnp.65.6.881

Renton, A. E., Majounie, E., Waite, A., Simón-Sánchez, J., Rollinson, S., Gibbs, J. R., et al. (2011). A hexanucleotide repeat expansion in C9ORF72 is the cause of chromosome 9p21-linked ALS-FTD. Neuron 72, 257-268. doi: 10.1016/j.neuron.2011.09.010

Reuter-Lorenz, P. A., and Cappell, K. A. (2008). Neurocognitive aging and the compensation hypothesis. Curr. Dir. Psychol. Sci. 17, 177-182. doi: $10.1111 / j .1467-8721.2008 .00570 . x$

Ringholz, G. M., Appel, S. H., Bradshaw, M., Cooke, N. A., Mosnik, D. M., and Schulz, P. E. (2005). Prevalence and patterns of cognitive impairment in sporadic ALS. Neurology 65, 586-590. doi: 10.1212/01.wnl.0000172911.39167.b6

Robberecht, W., and Philips, T. (2013). The changing scene of amyotrophic lateral sclerosis. Nat. Rev. Neurosci. 14, 248-264. doi: 10.1038/nrn3430

Roggeveen, A. B., Prime, D. J., and Ward, L. M. (2007). Lateralized readiness potentials reveal motor slowing in the aging brain. J. Gerontol. B. Psychol. Sci. Soc. Sci. 62, 78-84. doi: 10.1093/geronb/62.2.p78

Rustamov, N., Rodriguez-Raecke, R., Timm, L., Agrawal, D., Dressler, D., Schrader, C., et al. (2013). Absence of congruency sequence effects reveals neurocognitive inflexibility in Parkinson's disease. Neuropsychologia 51, 2976-2987. doi: 10.1016/j.neuropsychologia.2013.10.025

Rustamov, N., Rodriguez-Raecke, R., Timm, L., Agrawal, D., Dressler, D., Schrader, C., et al. (2014). Attention shifting in Parkinson's disease: an analysis of behavioral and cortical responses. Neuropsychology 28, 929-944. doi: 10.1037/neu0000099

Sarro, L., Agosta, F., Canu, E., Riva, N., Prelle, A., Copetti, M., et al. (2011). Cognitive functions and white matter tract damage in amyotrophic lateral sclerosis: a diffusion tensor tractography study. Am. J. Neuroradiol. 32, 1866-1872. doi: 10.3174/ajnr.A2658

Schoenfeld, M. A., Tempelmann, C., Gaul, C., Kühnel, G. R., Düzel, E., Hopf, J.-M., et al. (2005). Functional motor compensation in amyotrophic lateral sclerosis. J. Neurol. 252, 944-952. doi: 10.1007/s00415-005-0787-y

Schretlen, D. J. (2010). Modified Wisconsin Card Sorting Test (M-WCST). Lutz, FL: Psychological Assessment Resources.

Stanton, B. R., Williams, V. C., Leigh, P. N., Williams, S. C. R., Blain, C. R. V., Jarosz, J. M., et al. (2006). Altered cortical activation during a motor task in ALS: evidence for involvement of central pathways. J. Neurol. 254, 1260-1267. doi: 10.1007/s00415-006-0513-4

Strong, M. J., Grace, G. M., Freedman, M., Lomen-Hoerth, C., Woolley, S., Goldstein, L. H., et al. (2009). Consensus criteria for the diagnosis of frontotemporal cognitive and behavioural syndromes in amyotrophic lateral sclerosis. Amyotroph. Lateral Scler. 10, 131-146. doi: $10.1080 / 17482960802654364$

Strong, M., and Rosenfeld, J. (2003). Amyotrophic lateral sclerosis: a review of current concepts. Amyotroph. Lateral Scler. 4, 136-143. doi: $10.1080 / 14660820310011250$

Swinnen, B., and Robberecht, W. (2014). The phenotypic variability of amyotrophic lateral sclerosis. Nat. Rev. Neurol. 10, 661-670. doi: 10.1038/ nrneurol.2014.184

Taylor, L. J., Brown, R. G., Tsermentseli, S., Al-Chalabi, A., Shaw, C. E., Ellis, C. M., et al. (2013). Is language impairment more common than executive dysfunction in amyotrophic lateral sclerosis? J. Neurol. Neurosurg. Psychiatry 84, 494-498. doi: 10.1136/jnnp-2012-303526

Thorns, J., Wieringa, B. M., Mohammadi, B., Hammer, A., Dengler, R., and Münte, T. F. (2010). Movement initiation and inhibition are impaired in amyotrophic lateral sclerosis. Exp. Neurol. 224, 389-394. doi: 10.1016/j.expneurol.2010.04.014

Tsermentseli, S., Leigh, P. N., and Goldstein, L. H. (2012). The anatomy of cognitive impairment in amyotrophic lateral sclerosis: more than frontal lobe dysfunction. Cortex 48, 166-182. doi: 10.1016/j.cortex.2011.02.004 
Turner, M. R., Agosta, F., Bede, P., Govind, V., Lulé, D., and Verstraete, E. (2012). Neuroimaging in amyotrophic lateral sclerosis. Biomark Med. 6, 319-337. doi: 10.2217/bmm.12.26

Turner, M. R., Hardiman, O., Benatar, M., Brooks, B. R., Chiò, A., de Carvalho, M., et al. (2013). Controversies and priorities in amyotrophic lateral sclerosis. Lancet Neurol. 12, 310-322. doi: 10.1016/S1474-4422(13)70036-X

Turner, M. R., and Swash, M. (2015). The expanding syndrome of amyotrophic lateral sclerosis: a clinical and molecular odyssey. J. Neurol. Neurosurg. Psychiatry 86, 667-673. doi: 10.1136/jnnp-2014-308946

Ulrich, R., and Miller, J. (2001). Using the jackknife-based scoring method for measuring LRP onset effects in factorial designs. Psychophysiology 38, 816-827. doi: $10.1111 / 1469-8986.3850816$

Vallesi, A., and Stuss, D. T. (2010). Excessive sub-threshold motor preparation for non-target stimuli in normal aging. Neuroimage 50, 1251-1257. doi: 10.1016/j.neuroimage.2010.01.022

Vossel, S., Mathys, C., Daunizeau, J., Bauer, M., Driver, J., Friston, K. J., et al. (2014). Spatial attention, precision, and Bayesian inference: a study of saccadic response speed. Cereb. Cortex 24, 1436-1450. doi: 10.1093/cercor/bhs418

Wijesekera, L. C., and Leigh, P. N. (2009). Amyotrophic lateral sclerosis. Orphanet. J. Rare Dis. 4:3. doi: 10.1186/1750-1172-4-3

Wild-Wall, N., Falkenstein, M., and Hohnsbein, J. (2008). Flanker interference in young and older participants as reflected in event-related potentials. Brain Res. 1211, 72-84. doi: 10.1016/j.brainres.2008.03.025

Witgert, M., Salamone, A. R., Strutt, A. M., Jawaid, A., Massman, P. J., Bradshaw, M., et al. (2010). Frontal-lobe mediated behavioral dysfunction in amyotrophic lateral sclerosis. Eur. J. Neurol. 17, 103-110. doi: 10.1111/j.14681331.2009.02801.x
Witiuk, K., Fernandez-Ruiz, J., McKee, R., Alahyane, N., Coe, B. C., Melanson, M., et al. (2014). Cognitive deterioration and functional compensation in ALS measured with fMRI using an inhibitory task. J. Neurosci. 34, 14260-14271. doi: 10.1523/JNEUROSCI.1111-14.2014

Yeung, N., Botvinick, M. M., and Cohen, J. D. (2004). The neural basis of error detection: conflict monitoring and the error-related negativity. Psychol. Rev. 111, 931-959. doi: 10.1037/0033-295X.111.4.931

Yordanova, J., Kolev, V., Hohnsbein, J., and Falkenstein, M. (2004). Sensorimotor slowing with ageing is mediated by a functional dysregulation of motor-generation processes: evidence from high-resolution event-related potentials. Brain 127, 351-362. doi: 10.1093/brain/awh042

Zalonis, I., Christidi, F., Paraskevas, G., Zabelis, T., Evdokimidis, I., and Kararizou, E. (2012). Can executive cognitive measures differentiate between patients with spinal- and bulbar-onset amyotrophic lateral sclerosis? Arch. Clin. Neuropsych. 27, 348-354. doi: 10.1093/arclin/acs031

Conflict of Interest Statement: The authors declare that the research was conducted in the absence of any commercial or financial relationships that could be construed as a potential conflict of interest.

Copyright (c) 2015 Seer, Fürkötter, Vogts, Lange, Abdulla, Dengler, Petri and Kopp. This is an open-access article distributed under the terms of the Creative Commons Attribution License (CC BY). The use, distribution or reproduction in other forums is permitted, provided the original author(s) or licensor are credited and that the original publication in this journal is cited, in accordance with accepted academic practice. No use, distribution or reproduction is permitted which does not comply with these terms. 